\title{
Neuronal Activity in Monkey Superior Colliculus Related to the Initiation of Saccadic Eye Movements
}

\author{
Michael C. Dorris, Martin Paré, and Douglas P. Munoz \\ Medical Research Council Group in Sensory-Motor Neuroscience, Department of Physiology, Queen's University, \\ Kingston, Ontario, Canada K7L 3N6
}

\begin{abstract}
The introduction of a temporal gap between the disappearance of an initially fixated target and the appearance of an eccentric saccadic target results in a general reduction of saccadic reaction times (SRTs) - the gap effect-and often in the production of express saccades, the latencies of which approach the conduction time of the shortest neural pathways from the retina to the eye muscles. We investigated saccade initiation by recording neuronal activity in the superior colliculus in monkeys performing the gap paradigm. Fixation-related neurons reduced their discharge rate during the gap period, regardless of the SRT. This reduction in activity is consistent with the hypothesized release of ocular fixation that facilitates premotor processes and may contribute to the gap effect. In addition to saccade-related discharges, many saccade-related neurons displayed phasic target-related responses and/or low-frequency preparatory
\end{abstract}

activity during the gap period. The level of this preparatory activity correlated with both SRT and express saccade occurrence when the saccade was made into the response field of the neuron. Evidence indicates that advanced motor preparation is required for express saccade generation, which may be subserved by specific increases in the preparatory activity of saccade-related neurons. Increased preparatory activity may allow the target-related responses to trigger short-latency express saccades directly. This study provides insights into the functional mechanism of saccade initiation and may be relevant to the generation of all voluntary motor responses.

Key words: saccade; oculomotor; reaction times; superior colliculus; monkey; fixation; motor preparation; express saccades; gap effect.
Saccades are rapid, conjugate eye movements used to look at visual targets. The time required to initiate a saccade to a suddenly appearing target generally exceeds the conduction time of the shortest neural pathways from the retina to the extraocular muscles (Carpenter, 1981). A portion of this delay can be eliminated if the initially foveated fixation point is extinguished before the target presentation. The reduction in saccadic reaction times (SRTs) afforded by this gap-the gap effect-occurs irrespective of subject, target location, and training (Fischer and Weber, 1993; Paré and Munoz, 1996). A related but separate phenomenon, facilitated by the gap, is the production of express saccades, the latencies of which approach the fastest time for visual information to reach the oculomotor system and to be translated into a rapid eye movement (Fischer and Weber, 1993; Paré and Munoz, 1996). Express saccades form a distinct mode in the distribution of SRTs that is different from that of longer-latency regular saccades. In addition, express saccades are not produced in all subjects (Fischer and Ramsperger, 1984), and familiarity with target locations is usually required before they occur (Fischer et al., 1984; Boch and Fischer, 1986; Sommer, 1994; Paré and Munoz, 1996).

Two separate mechanisms have been hypothesized to account for the gap effect and express saccade occurrence (for detailed

Received June 3, 1997; revised Aug. 11, 1997; accepted Aug. 18, 1997.

This work was supported by the Medical Research Council of Canada. D.P.M. is a research scholar of the EJLB Foundation. We thank D. Hamburger and A. Lablans for technical assistance, P. Istvan, who participated in some of these experiments, and D. Hanes for supplying the algorithm for the Poisson spike train analysis.

Correspondence should be addressed to Douglas P. Munoz, Department of Physiology, Queen's University, Kingston, Ontario, Canada K7L 3N6.

Copyright (C) 1997 Society for Neuroscience $0270-6474 / 97 / 178566-14 \$ 05.00 / 0$ discussion, see Paré and Munoz, 1996). Of major contention is that the disengagement of ocular fixation hypothesis (ReuterLorenz et al., 1991; Munoz and Wurtz, 1992, 1993b; Kingstone and Klein, 1993b; Sommer, 1994; Tam and Ono, 1994; Dorris and Munoz, 1995) can account for the general SRT reduction of the gap effect but not for the spatial selectivity of express saccades. The latter is more readily explained by an oculomotor preparation hypothesis (Becker, 1989; Kowler, 1990; Paré and Munoz, 1996) which contends that topographically organized saccadic programs can be partially prepared before target presentation.

The main goal of this study is to understand the neural basis of SRTs and to distinguish between the two hypotheses of express saccade generation. We used extracellular recording techniques to measure changes in neuronal activity before target appearance in the monkey superior colliculus (SC), a structure hypothesized to be involved in saccade initiation. Collicular saccade-related neurons discharge a discrete highfrequency burst of action potentials for saccades to a restricted region of the visual field and often respond to the presentation of visual stimuli (Wurtz and Goldberg, 1972; Sparks et al., 1976; Sparks, 1978; Munoz and Wurtz, 1995a). A subset of saccade-related neurons additionally displays low-frequency discharges during the gap period preceding target appearance (Munoz and Wurtz, 1995a), which could represent advanced motor preparation. Fixation-related neurons, located at the rostral pole of the SC, are tonically active during periods of visual fixation and pause for saccades (Munoz and Wurtz, 1993a), and their activity is attenuated during the gap period (Dorris and Munoz, 1995) in a manner consistent with a disengagement of ocular fixation. Evidence from pharmacolog- 
ical (Hikosaka and Wurtz, 1985, 1986; Schiller et al., 1987; Munoz and Wurtz, 1993b) and microstimulation (Munoz and Wurtz, 1993b; Paré et al., 1994; Stanford et al., 1996) studies indicates that SRT (or the latency of electrically evoked saccades) is inversely related to the activation of saccade-related neurons and directly related to the activation of fixationrelated neurons. Of importance here, the ablation of the SC abolishes the production of express saccades (Schiller et al., 1987). To determine how the SC influences SRTs, we analyzed the activity of SC fixation- and saccade-related neurons recorded in the gap paradigm with respect to both SRTs and saccade initiation modes.

Some of these results have been reported in preliminary form elsewhere (Dorris and Munoz, 1995; Dorris et al., 1995).

\section{MATERIALS AND METHODS}

Animal preparation. We recorded the extracellular activity of single neurons in the intermediate layers of the SCs of two male rhesus monkeys (Macaca mulatta) weighing 5-6 kg each. All procedures were approved by the Queen's University Animal Care Committee and complied with the guidelines of the Canadian Council on Animal Care. Animals were under the close supervision of the university veterinarian.

Each monkey underwent a single surgical session to prepare for chronic recording of eye position and single neurons (Paré and Munoz, 1996). Eye coils were implanted subconjunctivally to measure eye position (Judge et al., 1980). Based on stereotaxic coordinates, two craniotomies were made to allow access to both SCs with microelectrodes. Stainless steel recording cylinders were positioned over the craniotomies, one centered on the midline and tilted $38^{\circ}$ posterior of vertical and the other centered on the interaural axis and tilted $25^{\circ}$ lateral of vertical.

Experimental procedures. Throughout the duration of the experiments, the monkeys were seated in a primate chair with their heads firmly attached to the chair via a head holder embedded in the explant. The monkeys faced a tangent screen $86 \mathrm{~cm}$ away, which spanned $\pm 35^{\circ}$ of the central visual field. Behavioral paradigms, visual displays, and storage of both neuronal discharge and eye movement data were under the control of a 486 personal computer running a real-time data acquisition system (REX) (Hays et al., 1982). REX controlled the presentation of the targets through digital to analog converters, which moved two mirror galvanometers (General Scanning) in orthogonal planes. These mirrors reflected a light-emitting diode $\left(2.0 \mathrm{~cd} / \mathrm{m}^{2}\right)$ on the translucent screen in front of the monkey. Eye movements were recorded with the magnetic search coil technique (Fuchs and Robinson, 1966), which had a resolution of $0.1^{\circ}$ (CNC Engineering). Horizontal and vertical eye and mirror positions were digitized at $500 \mathrm{~Hz}$. All data analysis was performed off-line.

The single-neuron activity was recorded with tungsten microelectrodes (Frederick Haer; 1-2 M $\Omega$ at $1 \mathrm{kHz}$ ), which were lowered through 23 gauge stainless steel guide tubes by a hydraulic microdrive (Narishige, Tokyo, Japan) attached to the recording chambers. The guide tubes were held firmly within a Delrin grid inside the recording chambers (Crist et al., 1988). Single-neuron discharges were sampled at $1 \mathrm{kHz}$ after passing through a window discriminator (Bak Electronics), which excluded action potentials that did not meet amplitude and time constraints.

Behavioral paradigms. Monkeys were initially trained to sit and drink water in the primate chair. They received a liquid reward when their eye position entered the invisible computer-controlled window surrounding a spot of light projected on the screen [hereafter referred to as the fixation point (FP)]. Over the ensuing days of training, the length of time of fixation was increased, whereas the size of the computer-controlled window was decreased. After learning to fixate for prolonged periods, the monkeys were trained to make a saccade to the spot of light when it stepped to an eccentric position (hereafter referred to as the target).

The monkeys were trained to perform the gap paradigm (Fig. 1). Trials were preceded by an intertrial interval (varied randomly between 500 and $1000 \mathrm{msec}$ ) during which the screen was illuminated with diffuse white light $\left(1.0 \mathrm{~cd} / \mathrm{m}^{2}\right)$ to prevent dark adaptation. The onset of a trial was signaled by the removal of this background light and appearance of the central FP. The monkey was required to look at the FP and to maintain fixation for 500-1000 msec. The FP was then extinguished, and there was a period (gap) during which the animal had to maintain fixation in total darkness before an eccentric target was presented. Within a block of trials, the gap duration was set at a constant duration of $200 \mathrm{msec}$ or

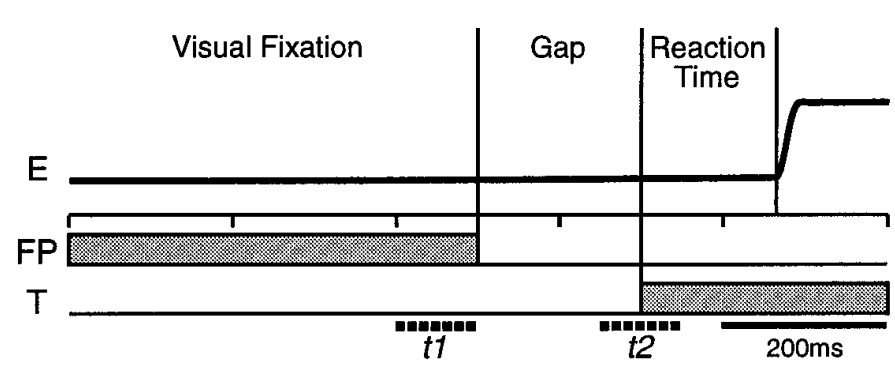

Figure 1. Schematic of the gap paradigm. Time is represented on the horizontal axis, and presentation of the visual stimuli ( FP, central fixation point; $T$, eccentric target) are denoted by the horizontal gray bars. As in the following figures, an upward deflection of the horizontal eye trace $(E)$ represents a rightward movement, and downward deflection represents a leftward movement. The visual fixation interval was randomized between 500 and $1000 \mathrm{msec}$, and the gap duration was either set at $200 \mathrm{msec}$ or randomized between 0 and $800 \mathrm{msec}$ within a block of trials. The discharge rate of neurons during this task was calculated during the final 100 msec of FP presentation (visual fixation epoch, t1) and from $50 \mathrm{msec}$ before to $50 \mathrm{msec}$ after target presentation (end of gap epoch, $t 2$ ).

randomized between 0 (no gap), 100, 200, 300, 400, 600, and $800 \mathrm{msec}$. The $200 \mathrm{msec}$ gap was chosen, because a significant percentage of express saccades are produced under this condition (Fischer and Boch, 1983; Schiller et al., 1987). After the target presentation, the monkey had 500 msec to make a saccade to the target and then to maintain fixation for $300-500 \mathrm{msec}$ before a liquid reward was given. Blocks of trials were run in which two possible target positions were randomly interleaved. For saccade-related neurons, one target was located contralateral to the side of the recording in the center of the response field of a neuron, whereas the other was equidistant from center on the opposite side of the horizontal and vertical meridians. For fixation-related neurons, which discharged optimally when targets were aligned with the fovea, targets were always presented randomly $10^{\circ}$ to the right or left of the FP. In a few cases, the target was presented only at the contralateral location.

In a separate block of 50-120 trials, the gap was fixed at $0 \mathrm{msec}$ (no gap), and target eccentricity was randomly interleaved at eight locations in the optimal direction to ascertain the optimal saccadic vector of each neuron and to determine its movement field characteristics using criteria described by Munoz and Wurtz (1995a). Typically, each block of trials consisted of the target being presented at the optimal direction and amplitude of the neuron, as well as two to four smaller and three to five larger amplitudes. For larger target eccentricities $\left(\geq 20^{\circ}\right)$, the FP was positioned on one side of the visual screen, and the target appeared on the opposite side. Thus, target steps of up to $70^{\circ}$ eccentricity in either the horizontal or the vertical plane were possible. The maximum amplitude tested for each neuron was at least $60^{\circ}$.

Once a neuron was isolated, and until either the isolation was lost or the series of paradigms was completed, the monkey usually performed the paradigms as follows: (1) the gap paradigm was run with a block of $200 \mathrm{msec}$ gap trials in an effort to obtain a sufficient sample of express and regular saccades; (2) the gap duration was randomized between 0 and $800 \mathrm{msec}$ to determine how neuronal activity evolved over longer gap durations; and (3) in the case of saccade-related neurons, 0 msec gap trials with target amplitude randomized along the optimal direction were run to determine the extent of the movement field of the neuron.

Data analysis. A Sun Sparc2 workstation was used to analyze the data. Computer software determined the beginning and end of each saccade using velocity and acceleration threshold and template-matching criteria (Waitzman et al., 1991). These events were verified by an experimenter to ensure accuracy. Rasters of neuronal discharge and continuously varying spike density functions (MacPherson and Aldridge, 1979; Richmond et al., 1987) were aligned on specific events in the paradigms. To generate the spike density function, a Gaussian pulse of a specified width was substituted for each spike, and then all Gaussians were summed together to produce a continuous function in time. The time from peak to $1 / e$ for each Gaussian was defined as $\sigma$. To analyze population responses during the gap, we convolved the spike train of each neuron with a Gaussian pulse width of $10 \mathrm{msec}$ to generate the spike density function for each gap duration. This value was chosen because it provided sufficient smoothing of the envelope of neuronal discharge without losing important details. The use of smaller Gaussian pulse (e.g., $4 \mathrm{msec}$ ) 


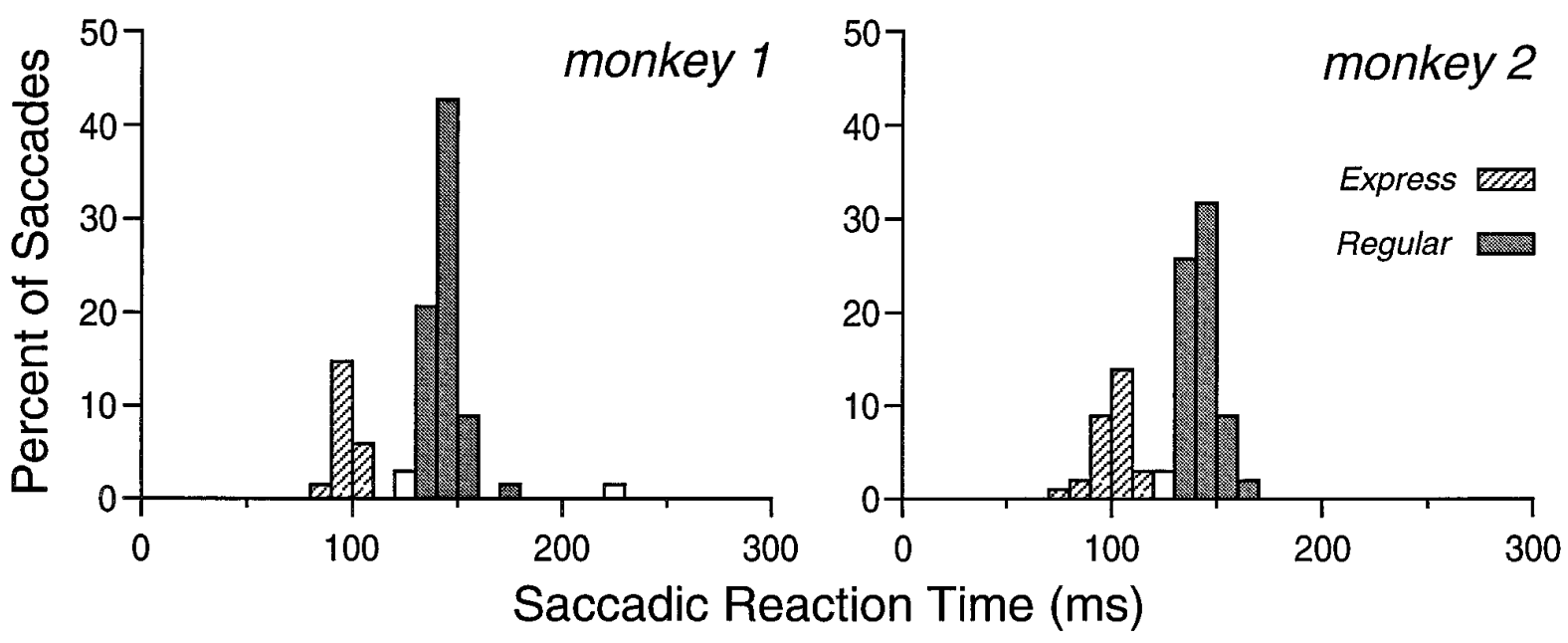

Figure 2. Typical SRT histograms obtained during a block of $200 \mathrm{msec}$ gap trials. Only the SRT distributions from one of two possible target locations is shown for each monkey. Hatched bars represent express saccades $(70-120 \mathrm{msec})$, and filled bars represent regular latency saccades $(130-180 \mathrm{msec})$.

added more noise to the shape of the spike density function without altering the overall shape.

To quantify the gap-related changes in neuronal activity, the discharge rate of individual neurons was measured during two different intervals in the gap paradigm: (1) the final $100 \mathrm{msec}$ before the FP was extinguished, while the monkey was fixating the FP (visual fixation epoch; Fig. 1, t1); and (2) the interval from $50 \mathrm{msec}$ before target appearance to $50 \mathrm{msec}$ after target appearance (end of gap epoch; Fig. 1, t2). All SC neurons in our sample that had target-related responses had response latencies $>50$ msec. Therefore, sampling during the $t 2$ epoch yielded a measure of activity immediately before any change that could be induced by the appearance of the eccentric target. This was a necessary prerequisite to test appropriately the hypotheses that express saccades are caused by changes in neuronal activity before target appearance related to either advanced motor preparation or disengagement of ocular fixation.

Trial-by-trial correlations (Pearson's $r$ ) between SRT and the discharge rate during these two epochs were calculated for each neuron. Two statistical methods were used to determine which factors, neuron type, epoch ( $t 1$ or $t 2)$, or target location (contralateral or ipsilateral), were involved in any correlations. First, the proportion of neurons with statistically significant correlation coefficients was calculated for each condition. Second, comparisons between the cumulative distributions of correlation coefficients were performed for each condition using a oneway ANOVA followed by an all-pair-wise multiple comparison procedure (Student-Newman-Keuls method).

To quantify the changes in activity preceding express and regular saccades, the level of activity during the visual fixation $(t 1)$ and end of gap $(t 2)$ epochs was computed separately for express and regular saccades. Examples of the SRT distributions during a block of $200 \mathrm{msec}$ gap trials for the two monkeys are shown in Figure 2. Based on the bimodality of the SRT distributions of the two monkeys, express saccades were defined arbitrarily as those initiated $70-120$ msec after target appearance, whereas regular saccades were defined as those initiated 130-180 msec after target appearance. This nomenclature is consistent with that developed by Fischer and colleagues (for review, see Fischer and Weber, 1993). Only neurons with at least five trials distributed within each of the express and regular saccade ranges were analyzed.

Edelman and Keller (1996) performed a detailed comparison of the activity of SC visuomotor burst neurons accompanying express and regular saccades. For express saccades, they noticed that these neurons discharged one burst of activity that was aligned with both target appearance and saccade onset. In contrast, the same neurons discharged two bursts for regular saccades, one target-aligned burst and a second saccade-aligned burst. They failed to reveal any significant difference between express and regular saccades in the latency to onset of the first burst. To determine whether there were differences between express and regular saccades in the latency to onset of the target-related burst of action potentials, we used a Poisson spike train analysis developed by Hanes et al. (1995) and Thompson et al. (1996). This analytical technique allowed us to distinguish the onset of bursts of action potentials from the mean discharge rate. We sampled the period from $700 \mathrm{msec}$ before (minimum $500 \mathrm{msec}$ visual fixation plus $200 \mathrm{msec}$ gap) to $200 \mathrm{msec}$ after (capturing both target- and saccade-related bursts) target appearance to obtain the mean discharge rate, which was used to generate a random Poisson distribution. The program identified in each trial the moment at which neural activity deviated maximally from that expected from a random Poisson distribution. Furthermore, only neurons showing targetrelated responses occurring from 50 to $100 \mathrm{msec}$ after target presentation for regular latency saccades were considered. This excluded neurons showing only a saccade-related response. Once again, only neurons with at least five trials of both express and regular saccades were considered in this analysis.

To determine the magnitude of target- and saccade-related bursts of action potentials exhibited by saccade-related neurons, the maximum value reached by spike density functions constructed from rasters aligned on the time of occurrence of the target and the saccade were used, respectively. The spike density functions were constructed from the average of rasters only from neurons with at least five trials of both express and regular saccades. Specifically, the target-related burst was considered the largest peak in the target-aligned spike density function that occurred between 60 and $120 \mathrm{msec}$ after target presentation. The saccade-related burst was considered the largest peak in the saccadealigned spike density function that occurred closest to the time of saccade initiation that was not more than $\pm 20 \mathrm{msec}$ from saccade initiation.

Neuron classification. We separated our neurons into three different classes of SC neurons based on the previous descriptions of Munoz and Wurtz (1993a) for fixation neurons and Munoz and Wurtz (1995a) for buildup and burst neurons. This classification was performed with the data obtained in the blocks of $200 \mathrm{msec}$ gap trials. To be classified as a fixation neuron, a neuron recorded in the SC rostral pole had to display (1) tonic activity of $>10$ spikes/sec during both the visual fixation $(t 1)$ and end of gap ( $t 2)$ epochs, i.e., while the monkey fixated the FP even when it was momentarily removed and was required to maintain the same eye position (this excluded simple visual neurons with a foveal receptive field); and (2) a pause in discharge when the monkey generated all ipsiversive saccades and most contraversive saccades. To be classified as a buildup neuron, a neuron had to display (1) long-lead prelude activity during the end of the gap $(t 2)$ epoch that was significantly greater than during the visual fixation $(t 1)$ epoch $(t$ test, $p<0.05)$; and (2) saccaderelated activity of $>100$ spikes/sec for saccades into the response field of the neuron. To be classified as a burst neuron, a neuron had to display (1) no significant increase in discharge from visual fixation $(t 1)$ to the end of the gap ( $t 2)$ epoch ( $t$ test, $p>0.05)$; and (2) saccade-related activity of $>100$ spikes/sec for saccades into the response field of the neuron. Consistent with the descriptions of Munoz and Wurtz (1995a), when the movement field of the neurons was tested extensively, nearly all buildup neurons (15 of 18) exhibited saccade-related activity associated with saccades in the same direction but of amplitude significantly larger than the optimal amplitude of the neuron (i.e., open-ended movement field), 


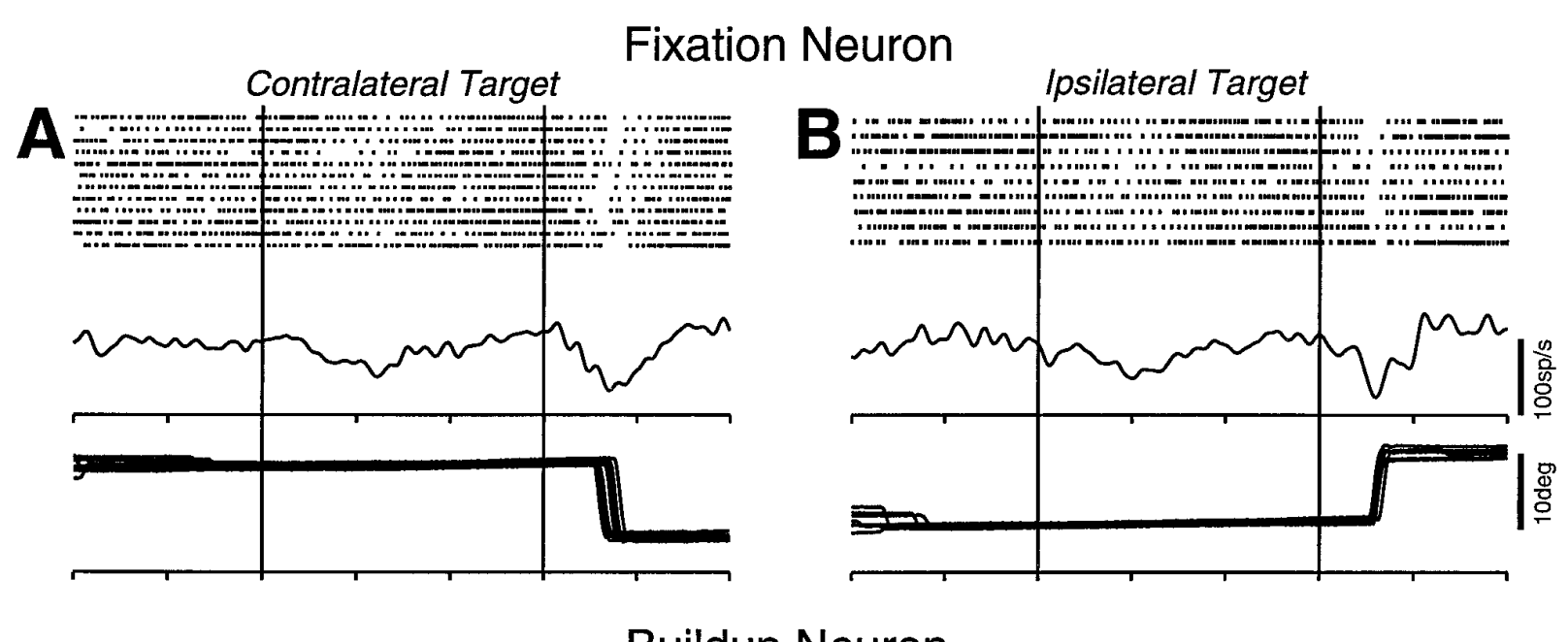

\section{Buildup Neuron}
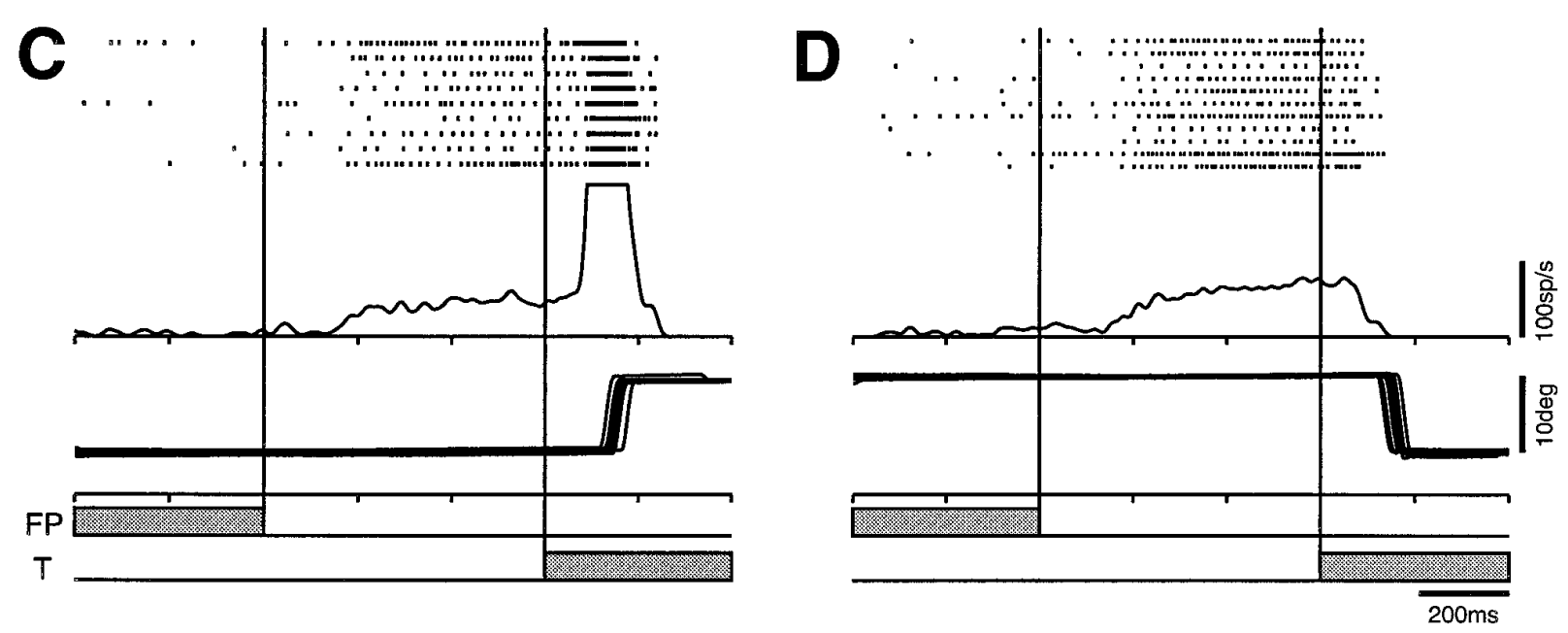

Figure 3. The activity of a single fixation $(A, B)$ and buildup $(C, D)$ neuron during $600 \mathrm{msec}$ gap trials. The fixation neuron was located in the right $\mathrm{SC}$, and the target was presented $10^{\circ}$ left $\left(A\right.$, contralateral target) or $10^{\circ}$ right $(B$, ipsilateral target). The buildup neuron was located in the left $\mathrm{SC}$, and the target was presented $10^{\circ}$ right $\left(C\right.$, contralateral target optimal vector for the response field of this neuron) or $10^{\circ}$ left $(D$, ipsilateral target opposite the response field of this neuron). Trials were collected in a block of trials in which gap duration was randomized between $0,100,200,300,400,600$, and $800 \mathrm{msec}$, and the target appeared randomly $10^{\circ}$ to the right or left. The individual rasters of neuron discharge, the spike density function, and the horizontal eye position traces are aligned on both FP disappearance (left vertical line) and target onset (right vertical line).

whereas most of the burst neurons (50 of 61) discharged for a narrow range of saccade amplitudes (i.e., closed movement field).

\section{RESULTS}

Of all the neurons recorded from both SC of two monkeys performing the gap paradigm, only 159 fulfilled our classification criteria and had a significant portion of the testing completed to be analyzed. According to our criteria, 29\% (46 of 159) were fixation, $19 \%$ (30 of 159) were buildup, and 52\% (83 of 159) were burst neurons.

\section{Gap-related neuronal activity}

The tonic activity of fixation neurons was modulated in a characteristic manner during the gap period (Dorris and Munoz, 1995). The activity of a single fixation neuron recorded in the rostral pole of the right SC is shown in Figure 3 for trials with a gap duration of $600 \mathrm{msec}$ and the target presented $10^{\circ}$ left (Fig. $3 A$, contralateral target) and $10^{\circ}$ right (Fig. $3 B$, ipsilateral target). The neuron was tonically active during visual fixation and displayed the characteristic pause in discharge associated with the generation of saccades. During the gap, the level of activity decreased and reached a minimum $\sim 250 \mathrm{msec}$ after FP disappearance. The activity level increased subsequently as the gap duration increased, achieving the level of activity that preceded the gap. Figure 4 shows the mean spike density functions from all fixation neurons for the $200 \mathrm{msec}$ (Fig. 4A) and $600 \mathrm{msec}$ (Fig. $4 B$ ) gap trials with contralateral target. During visual fixation, the mean discharge rate of fixation neurons was the highest. The discharge rate of the fixation neurons decreased shortly $(\sim 100$ msec) after FP disappearance and was lowest $\sim 200-300 \mathrm{msec}$ into the gap. With the longer gap durations (Fig. 4B), the activity of many of the neurons increased, although the average population activity did not recover to the level achieved before FP disappearance. Some neurons had a transient increase in discharge occurring 70-90 msec after FP disappearance.

The activity of a single buildup neuron recorded in the left SC is shown in Figure 3 for $600 \mathrm{msec}$ gap trials and the target presented $10^{\circ}$ right (Fig. $3 C$, contralateral target) and $10^{\circ}$ left (Fig. $3 D$, ipsilateral target). The neuron had very little activity while 
Figure 4. Reciprocal pattern of activity for the sample of fixation and buildup neurons during fixed $200 \mathrm{msec}$ (46 fixation and 30 buildup neurons) $(A)$ and randomized $600 \mathrm{msec}(B)$ gap trials (31 fixation and 15 buildup neurons). The thick line represents the mean spike density, and the envelope surrounding each waveform represents the SEM.

Figure 5. Mean discharge rate of individual fixation $(A)$ and buildup $(B)$ neurons during the visual fixation $(t 1)$ and end of gap ( $t 2)$ epochs during $200 \mathrm{msec}$ gap trials. Each data point represents a single neuron. Lines of equality are represented by dashed lines, and linear regression lines are represented as solid lines.
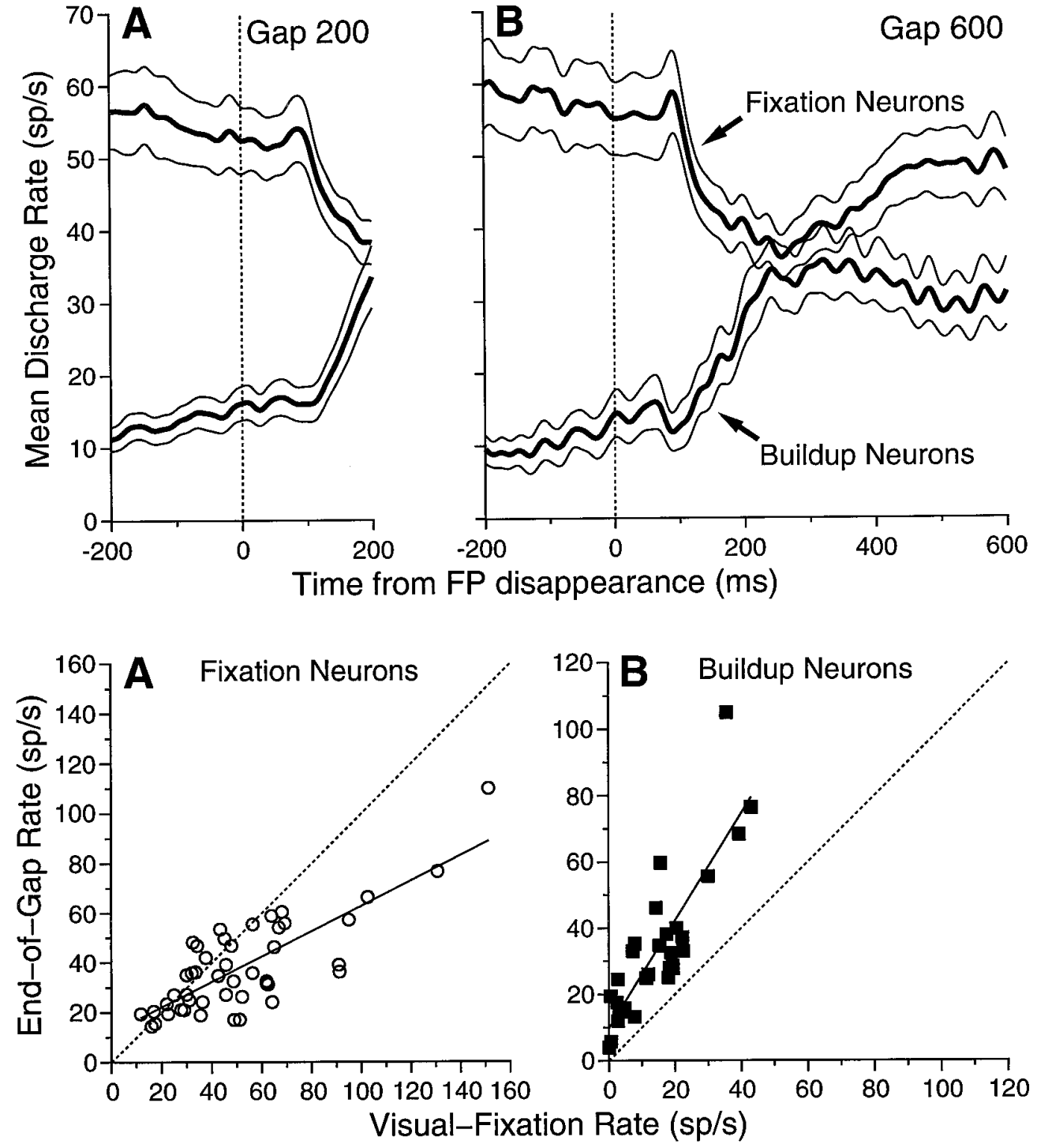

the monkey was fixating the FP. During the gap, an increase in neuronal activity began slightly more than $100 \mathrm{msec}$ after the FP disappeared. This discharge remained at a tonic level until target appearance, when a burst of activity occurred for saccades of the optimal vector (Fig. $3 C$ ), or the activity was truncated for saccades in the opposite direction (Fig. 3D). The pattern of activity of buildup neurons during the gap period was reciprocal to that recorded from fixation neurons (Fig. 4A,B). Before disappearance of the FP, the mean discharge rate of the buildup neurons was low, averaging 10 spikes/sec. Approximately $100 \mathrm{msec}$ after FP disappearance the activity of buildup neurons began to increase abruptly. After $\sim 250 \mathrm{msec}$ into the gap, buildup neurons reached their peak level of discharge, which decreased slightly with increasing gap duration (Fig. 4B).

The distribution of mean discharge rates of individual fixation and buildup neurons during the visual fixation $(t 1)$ and end of gap $(t 2)$ epochs is illustrated in Figure 5. Most fixation neurons decreased their discharge during the gap, and greater activity during visual fixation was generally associated with greater activity at the end of the gap $(n=46$; slope $=0.51 ; r=0.79 ; p<0.001)$ (Fig. $5 A$ ). By definition, all buildup neurons increased their discharge during the gap, but the level of activity reached at the end of the gap was also related to that observed during visual fixation $(n=30$; slope $=1.62 ; r=0.85 ; p<0.001)$ (Fig. $5 B)$.

\section{Relationship between SRT and neuronal activity}

We investigated whether specific measures of the neuronal activity of fixation and buildup neurons before or during the gap period was predictive of SRT on a trial-by-trial basis. Because the activity of both fixation and buildup neurons was modulated with gap duration (see Fig. 4), randomizing gap duration could act as a confounding variable. In addition, the effects on neuronal activity of the presence of the FP in trials with a short gap duration (i.e., 0 and $100 \mathrm{msec}$ ) introduced an uncontrolled variable. This analysis was therefore performed in two ways. First, analysis was performed using data collected from blocks of trials in which the gap was maintained at a constant duration of $200 \mathrm{msec}$. Second, the effects of different gap durations on data analysis were considered separately by examining the SRT in blocks of trials in which the gap duration was randomized between 0 and $800 \mathrm{msec}$. The discharge rate was calculated during the $t 1$ and $t 2$ epochs for each trial and was plotted against the corresponding SRT for that trial. A minimum of 12 trials for a neuron was used in this analysis, although the majority of neurons had between 30 and 
Fixation Neuron

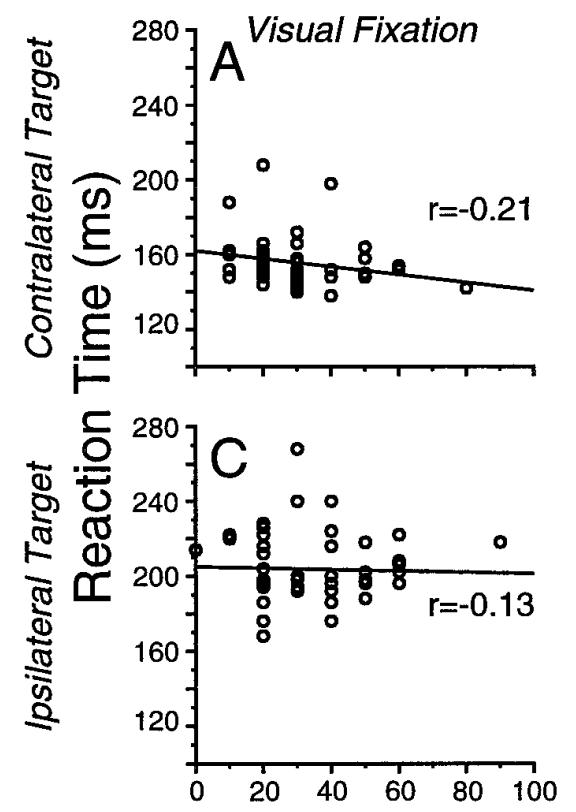

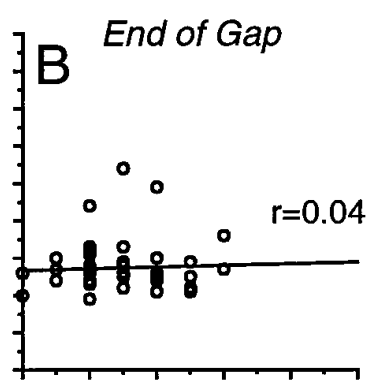
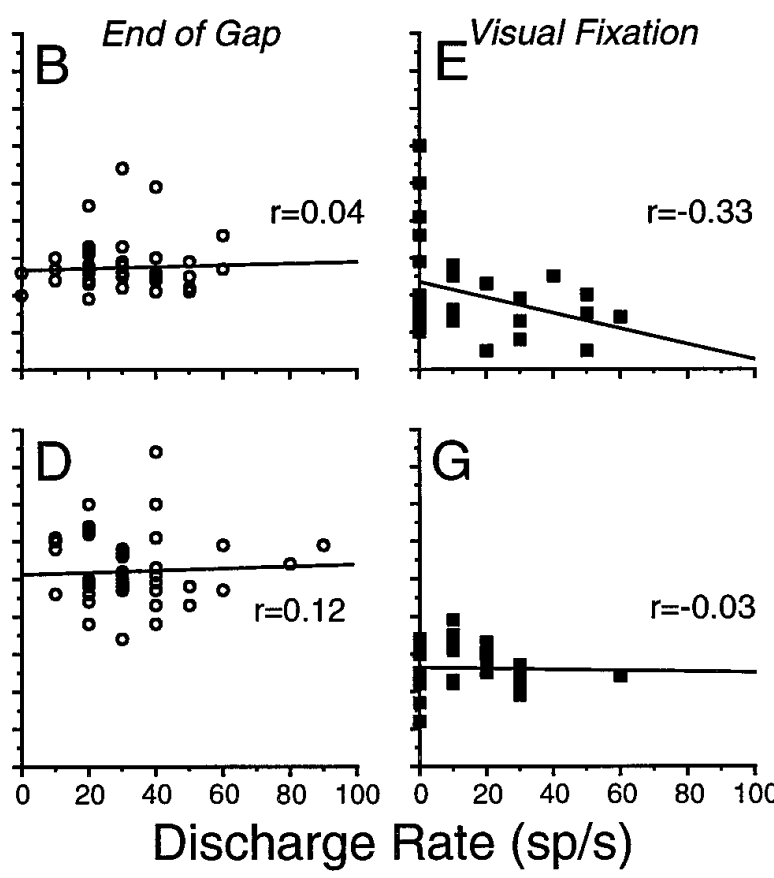
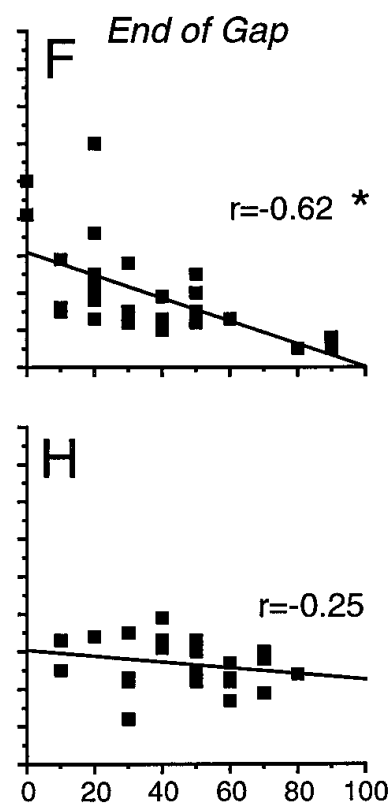

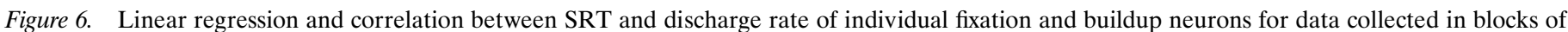

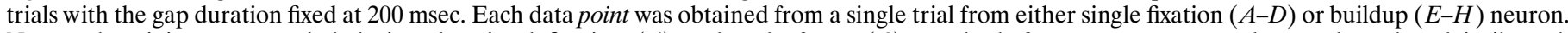

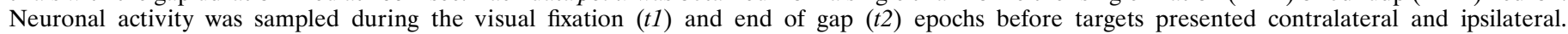
*Statistically significant correlation $(p<0.05)$.

Table 1. Percentage of neurons with significant correlation coefficients between SRT and neuronal discharge

\begin{tabular}{|c|c|c|c|c|c|}
\hline \multirow[b]{2}{*}{ Neuron } & \multirow[b]{2}{*}{ Gap (msec) } & \multicolumn{2}{|c|}{ Contralateral target } & \multicolumn{2}{|c|}{ Ipsilateral target } \\
\hline & & $t 1$ & $t 2$ & $t 1$ & $t 2$ \\
\hline \multirow[t]{2}{*}{ Fixation } & $200 \mathrm{msec}$ & $11 \%(5 / 44)$ & $14 \%(6 / 44)$ & $5 \%(2 / 40)$ & $10 \%(4 / 40)$ \\
\hline & Random & $16 \%(5 / 31)$ & $13 \%(4 / 31)$ & $23 \%(7 / 31)$ & $16 \%(5 / 31)$ \\
\hline \multirow[t]{2}{*}{ Buildup } & $200 \mathrm{msec}$ & $14 \%(4 / 29)$ & $41 \%(12 / 29)$ & $11 \%(2 / 19)$ & $11 \%(2 / 19)$ \\
\hline & Random & $33 \%(5 / 15)$ & $53 \%(8 / 15)$ & $18 \%(2 / 11)$ & $23 \%(3 / 11)$ \\
\hline
\end{tabular}

100 trials. Using linear regression analysis of the relationship between SRT and discharge rate, correlation coefficients were calculated for each individual neuron, for the two epochs, for the contralateral and (when possible) ipsilateral targets.

We first describe the results of the analysis of the data collected from blocks of $200 \mathrm{msec}$ gap duration trials. Examples of the relationships and correlations obtained for one fixation and one buildup neuron for $200 \mathrm{msec}$ gap duration trials are illustrated in Figure 6. No significant relationship existed between SRT and discharge rate during the visual fixation $(t 1)$ epoch for any of the conditions in either of the two neurons shown $(p>0.05)$ (Fig. $6 A, C, E, G)$. Nor did a significant relationship exist between SRT and the discharge rate during the end of the gap ( $t 2)$ epoch (Fig. $6 B, D, H)$, with the exception of the buildup neuron activity associated with the contralateral target $(r=-0.62 ; p<0.001)$ (Fig. $6 F$ ). In this case, the greater the activity, the shorter the SRT.

Table 1 shows the proportion of fixation and buildup neurons that had significant correlations $(p<0.05)$ between SRT and discharge rate. For the data collected from blocks of trials with a constant gap duration of $200 \mathrm{msec}$, the neuronal activity of fixation neurons was not well correlated for any of the epochs or target location tested; $<15 \%$ of the neurons showed significant correlations in any condition. Buildup neuron activity was also poorly correlated with SRT, except at the end of gap ( $t 2)$ epoch before saccades into their contralateral response field. In this condition, $41 \%$ (12 of 29) of the buildup neurons had discharge rates that were significantly correlated to SRT. A factor that may have limited the proportion of neurons showing a significant correlation between SRT and mean discharge rate was the limited distribution of SRTs obtained during many recordings. For some neurons, the distribution of SRTs obtained was unimodal with very little variance. Also summarized in Table 1 is the percentage of fixation and buildup neurons having significant correlations between SRT and discharge rate in the $t 1$ and $t 2$ epochs with the data set obtained from randomized gap trials of $0-800 \mathrm{msec}$. This data set usually contained a wider range of SRTs and a greater number of trials. The neuronal activity of fixation neurons was not well correlated for any of the epochs or target locations tested. Although the percentage of significant correlations increased with respect to the results of the analysis restricted to the 200 msec gap trials, it never reached $25 \%$ in any condition. Buildup neuron activity was also poorly correlated with SRT, except 
before saccades into their contralateral response field. In this condition, $33 \%$ (5 of 15) of the buildup neurons had discharge rates that were significantly correlated to SRT for the $t 1$ epoch, and $53 \%$ ( 8 of 15 ) had significant correlations for the $t 2$ epoch.

Another method to isolate which, if any, of the combinations of neuron types, target locations, and epoch discharge rates are most related to SRT compares distributions of cumulative correlation coefficients (adapted from Riehle and Requin, 1993). Figure 7 shows the results of this analysis for the data collected from the blocks of trials with only 200 msec gap duration. Each data point in Figure 7 represents the correlation coefficient between SRT and discharge rate from one neuron, such as computed in Figure 6. If two of these cumulative correlation coefficient curves overlap closely, it suggests that the activities of the two populations are equally correlated to SRT. If, however, one curve is shifted further from zero than another curve, it suggests that the activity of the population constituting the curve more distant from zero is more correlated to SRT than the population constituting the more central curve.

Figure $7 A$ shows the cumulative correlation coefficient distributions for fixation neurons for two epochs ( $t 1$ or $t 2)$ and two target locations (contralateral or ipsilateral). All four curves closely overlap, suggesting that neither direction of the target nor the epoch influenced the correlation between SRT and the discharge rate of these neurons. There was no significant difference among these fixation neuron curves (ANOVA, $p>0.05$ ). Furthermore, the four mean values of these fixation neuron distributions varied little from zero (Table 2), further demonstrating the lack of predictive value of fixation neuron activity for SRT. The cumulative correlation coefficient distributions constructed for buildup neurons (Fig. $7 B$ ) did not differ from one another (Student-Newman-Keuls method, $p>0.05$ ) with the exception of the buildup neuron curve for the $t 2$ epoch when the target was presented at the contralateral location (Student-Newman-Keuls method, $p<0.05)$. For the three conditions in which the distributions did not differ, the three mean values did not vary much from zero (Table 2), whereas the curve constructed from the buildup neuron correlation coefficients from the $t 2$ epoch and the contralateral target had a mean correlation value of -0.23 . Among the neurons with significant correlations, the mean was -0.49 (range, -0.26 to -0.71 ).

It is not surprising that the most important epoch in which neuronal activity can influence SRT is the end of gap ( $t 2)$ epoch just before target presentation. This epoch represents the level of neuronal activity just before visual target information reaching the SC. Figure $7 C$ compares the correlation values of buildup and fixation neurons during the $t 2$ epoch when the target is presented in the contralateral direction. This further exemplifies the degree to which buildup activity is a better predictor of upcoming SRT with its curve being clearly shifted from zero compared with that of fixation neurons ( $t$ test, $d f=71 ; t=3.59 ; p<0.001$ ).

\section{Neuronal activity and express saccades}

We have shown that the activity of fixation neurons and buildup neurons was modulated during the gap period. However, SRT was best reflected by the activity of buildup neurons during the end of gap ( $t 2)$ epoch before saccades into the response field of the neuron. We now ask the more specific question of whether, before the appearance of the target, the activity of either of these neuron populations is related to the occurrence of express saccades produced in the gap paradigm. According to the fixation disengagement and oculomotor preparation hypotheses, the ac-

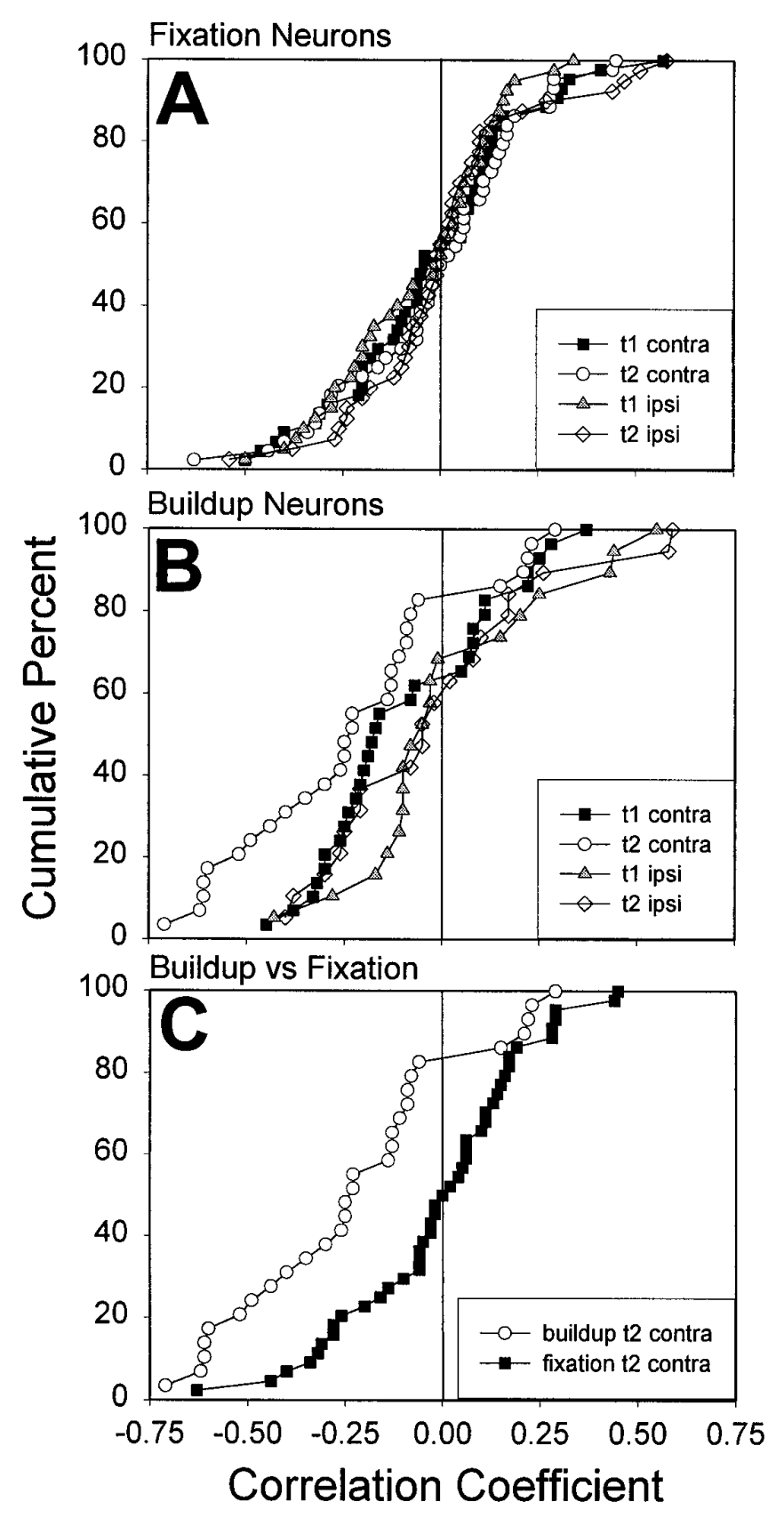

Figure 7. Correlation coefficients between SRT and discharge rate of individual neurons plotted as a cumulative distribution. All data were collected from blocks of trials with the gap duration fixed at $200 \mathrm{msec}$. Each data point represents the correlation coefficient from one neuron when the activity was sampled during different epochs or target locations. $A$, The fixation neuron distributions were centered around zero and did not differ from each other (ANOVA, $p>0.05$ ). $B$, The distribution made from buildup neuron activity during the $t 2$ epoch and for the contralateral target was shifted to the left of zero and differed from the other buildup neuron distributions (ANOVA, Student-Newman-Keuls method, $p<$ 0.05). $C$, The distribution composed from the correlation coefficients of the $t 2$ contralateral target buildup neurons differed significantly from the distribution composed of the $t 2$ contralateral target fixation neuron distribution $(t$ test, $p<0.01)$.

tivity of fixation and buildup neurons, respectively, should be correlated to the generation of express saccades through their effect on the saccade-generating circuit before target presentation. To test these hypotheses, the activity of individual neurons 
Table 2. Means of correlation coefficients between SRT and neuronal discharge rate

\begin{tabular}{|c|c|c|c|c|c|}
\hline \multirow[b]{2}{*}{ Neuron } & \multirow[b]{2}{*}{ Gap (msec) } & \multicolumn{2}{|c|}{ Contralateral target } & \multicolumn{2}{|c|}{$\underline{\text { Ipsilateral target }}$} \\
\hline & & $t 1$ & $t 2$ & $t 1$ & $t 2$ \\
\hline Fixation & 200 & -0.02 & -0.01 & -0.06 & 0.01 \\
\hline Buildup & 200 & -0.09 & -0.23 & 0.02 & -0.01 \\
\hline
\end{tabular}

\section{A Fixation Neuron B Buildup Neuron}
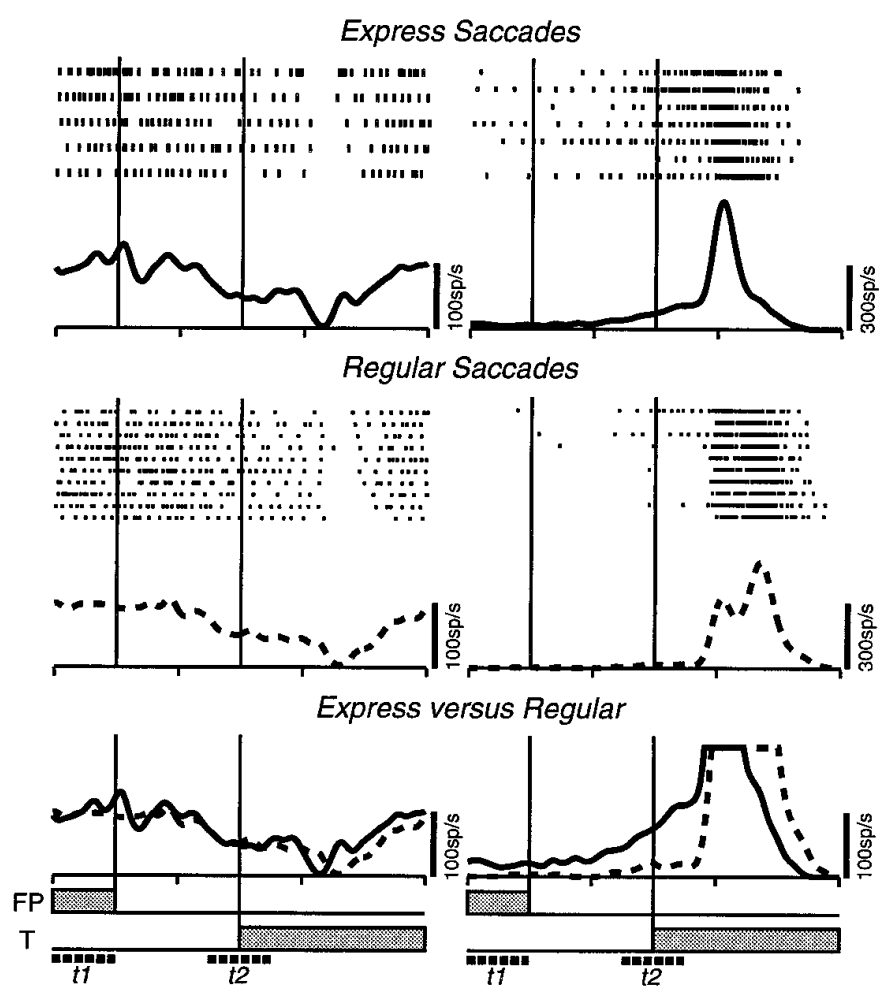

Figure 8. Comparison of neuronal discharge during the generation of express and regular saccades in $200 \mathrm{msec}$ gap trials. The spike density functions generated from express (top, solid line) and regular (middle, dashed line) trials are aligned on target appearance and superimposed at the bottom. $A$, The spike density functions constructed from a fixation neuron did not differ $(t$ test, $p>0.05)$. B , The end of gap ( $t 2)$ discharge rate of the buildup neuron was greater before express saccade generation compared with the generation of regular saccades ( $t$ test, $p<0.005)$. When the scale is expanded to 300 spikes/sec, the spike density function generated for regular saccades displayed two peaks: one burst time locked to the appearance of the visual stimulus and a later burst time locked to the generation of the saccade. The spike density function for express saccades had only one robust peak time locked to both target and saccade onsets.

recorded during the constant 200 msec gap trials was segregated on the basis of whether the SRTs were of express or regular latency.

Target-aligned rasters and spike density functions of the activity of a fixation neuron are shown in Figure $8 A$ for the generation of express (top) and regular saccades (middle). The typical gaprelated decrease in fixation neuron activity beginning $\sim 100 \mathrm{msec}$ into the gap was observed for both types of saccades. The two spike density functions are superimposed at the bottom of Figure $8 A$, and, except for the earlier pause in activity associated with the shorter latency express saccades, there was little difference

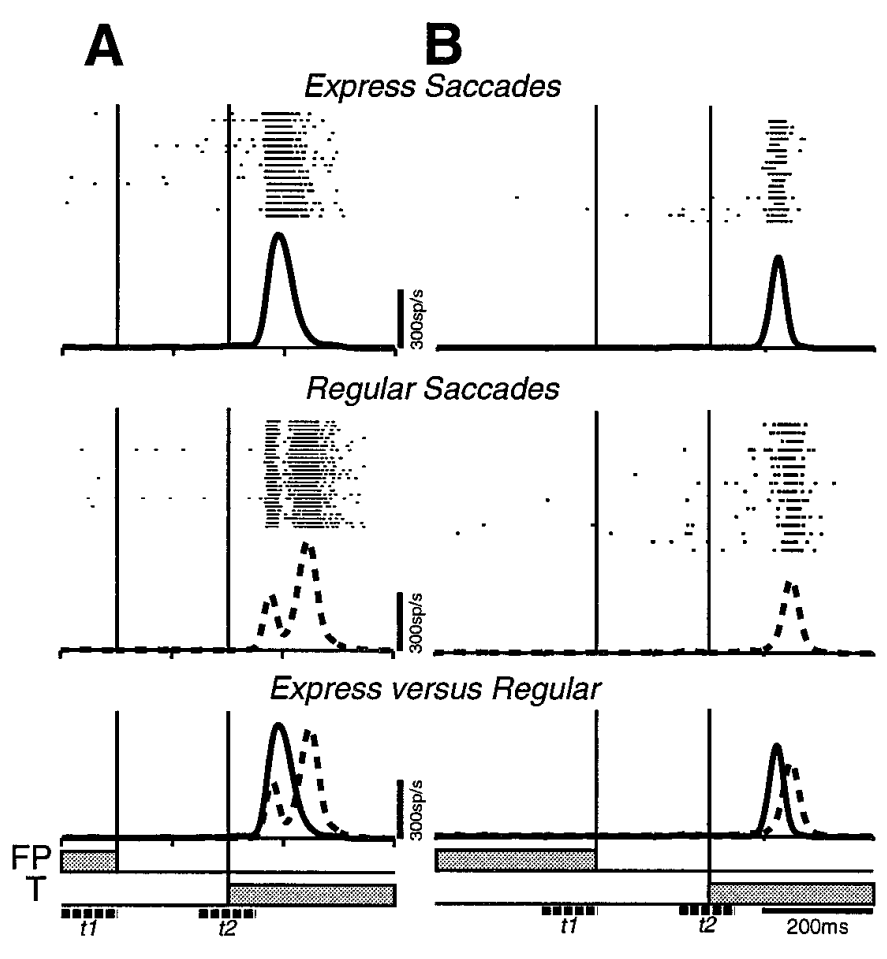

Figure 9. Comparison of neuronal discharge during the generation of express and regular saccades in $200 \mathrm{msec}$ gap trials for burst neurons. The spike density functions generated from express (top, solid line) and regular (middle, dashed line) trials are aligned on target appearance and superimposed at the bottom. $A$, Spike density functions constructed from a burst neuron with target-related activity. $B$, Spike density functions constructed from a burst neuron without target-related activity.

between them at either the $t 1$ or $t 2$ epoch ( $t$ test, $p>0.05)$. In contrast, the gap-related discharge rate of the buildup neuron was much higher before express saccades than before regular saccades, as shown by the superposition of spike density functions (Fig. $8 B$ ). This was quantified by measuring the activity at the end of the gap ( $\mathrm{t} 2$ ) epoch ( $t$ test, $d f=18 ; t=3.56 ; p<0.005$ ). In addition, after target appearance, the buildup neuron discharged only one burst of action potentials for express saccades but two bursts for regular saccades. During regular saccade trials, the first burst began $\sim 70 \mathrm{msec}$ after target appearance, whereas the second burst was aligned with the occurrence of the saccade. During express saccade trials, the single burst was aligned with both target appearance and saccade occurrence. The merging of the two bursts during express saccades was also observed for those burst neurons showing two bursts of action potentials for regular saccades (Fig. 9A). This latter finding corroborates observations of visuomotor burst neurons previously reported by Edelman and Keller (1996). Buildup and burst neurons lacking target-related responses nevertheless discharged a burst of action potentials for express saccades (Fig. 9B).

From our sample, 24 fixation neurons and 17 buildup neurons 


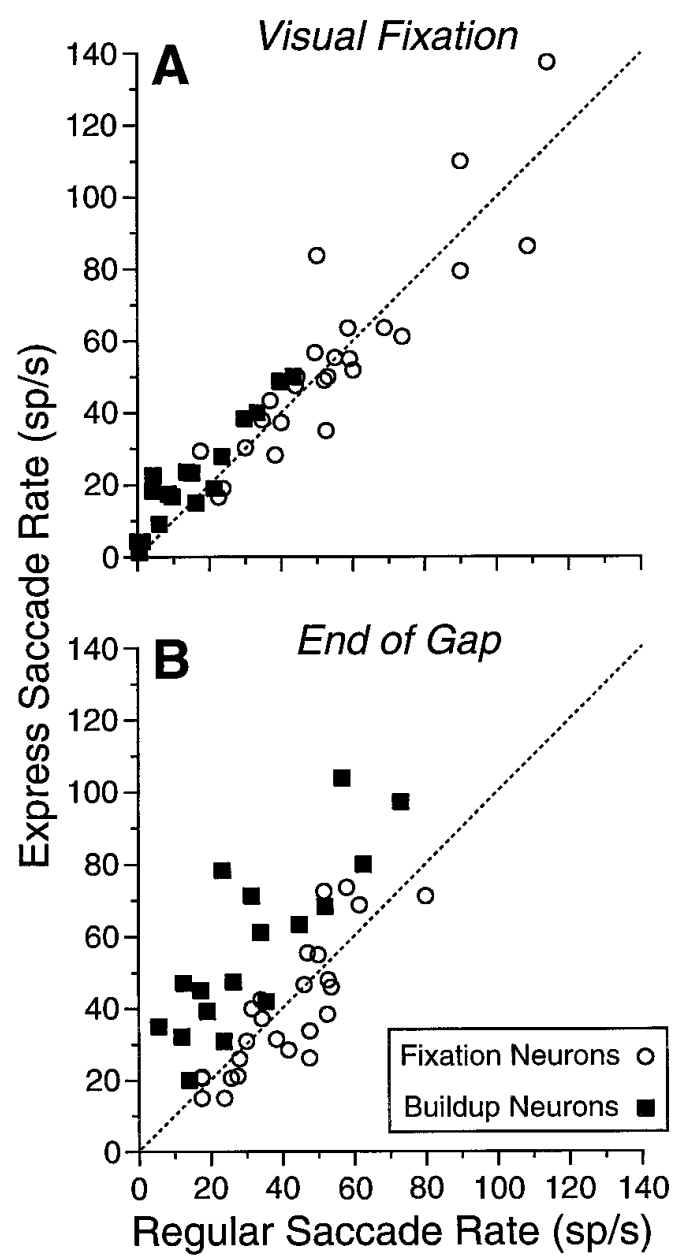

Figure 10. Mean discharge rate before express and regular saccades of 17 buildup neurons and 24 fixation neurons during the visual fixation $(t 1$; $A)$ and the end of gap $(t 2 ; B)$ epochs. The dotted line represents the equality line.

could be analyzed quantitatively (minimum of five trials of both express and regular saccades). Figure 10 shows the individual discharge rates of these neurons associated with express and regular saccades during visual fixation ( $t 1)$ and end of gap ( $t 2)$ epochs. Figure 11 contrasts the mean discharge rates for the sample of neurons. Data obtained from 41 burst neurons, the gap-related discharges of which were usually minimal (Fig. 9), are also presented in Figure 11. During the visual fixation ( $t 1)$ epoch, discharge rates of most fixation and buildup neurons preceding express and regular saccades were similar, being distributed around the line of equality (Fig. 10A). The mean discharge rate \pm SEM of fixation neurons (Fig. 11 $A$ ) was $55 \pm 6$ (range, 17-138) spikes/sec before express saccades and $55 \pm 5$ (range, 17-114) spikes/sec before regular saccades (paired $t$ test, $d f=23 ; t=$ 0.164; $p=0.87$ ). Furthermore, no individual fixation neuron had significantly different discharge rates preceding express and regular saccades ( $t$ test, $p>0.05$ ). The mean discharge rate of buildup neurons (Fig. 11C) was $22 \pm 4$ (range, 1-50) spikes/sec before express saccades and $16 \pm 3$ (range, 0-43) spikes/sec before regular saccades. This small difference was statistically significant (paired $t$ test, $d f=16 ; t=5.10 ; p<0.0001$ ). Among individual buildup neurons, 3 of 17 had a significantly higher rate of discharge preceding express saccades ( $t$ test, $p<0.05)$. The
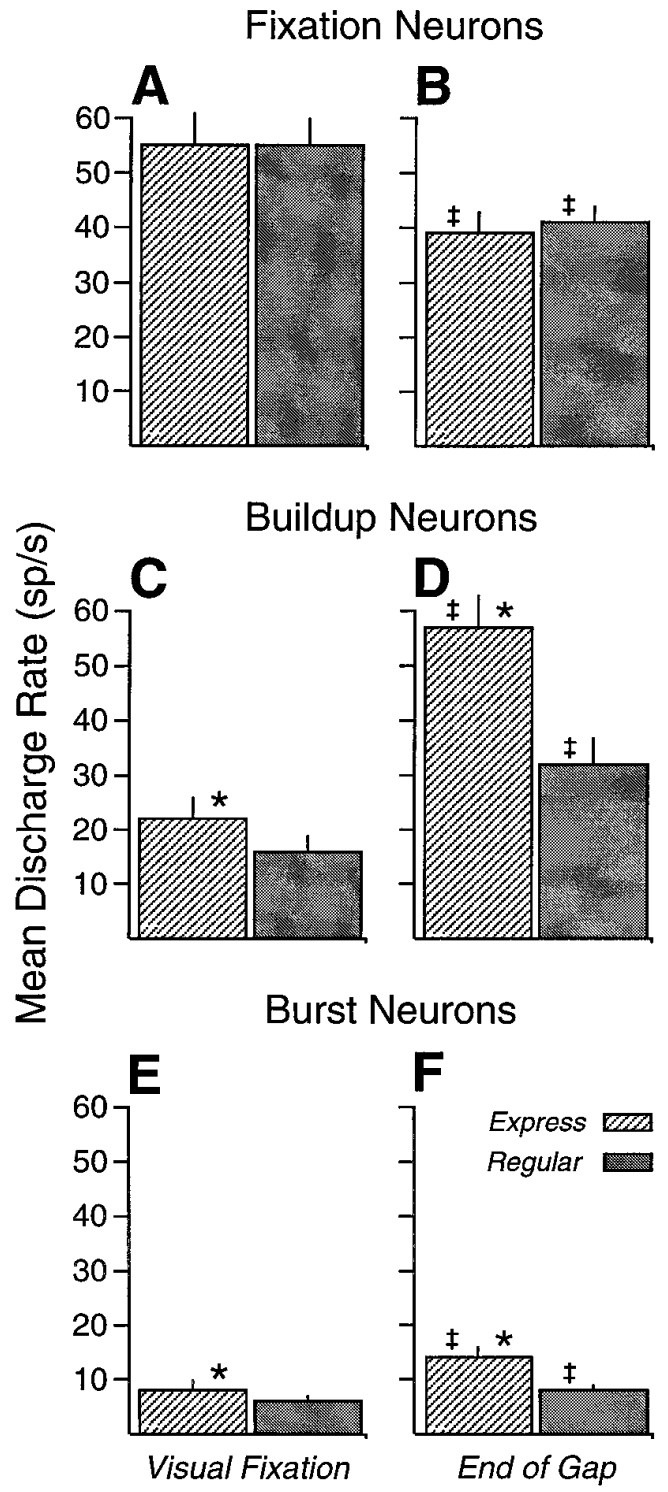

Figure 11. Mean discharge rate before express and regular saccades for the population of fixation $(A, B)$, buildup $(C, D)$, and burst $(E, F)$ neurons during visual fixation $(t 1)(A, C, E)$ and the end of gap epochs $(t 2)(B, D$,

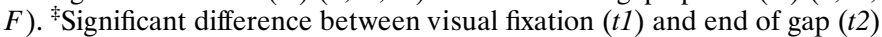
epochs. *Significant difference between express and regular saccades within a sampling period.

mean discharge rate of burst neurons during the visual fixation $(t 1)$ epoch (Fig. 11E) was $8 \pm 2$ (range, 0-33) spikes/sec before express saccades and $6 \pm 1$ (range, 0-26) spikes/sec before regular saccades. This small difference was statistically significant (Wilcoxon signed rank test, $p<0.01$ ). Thus, during the visual fixation $(t 1)$ epoch, the mode of saccade initiation could not be predicted on the basis of activity of fixation neurons, and the predictive capability of buildup and burst neurons in terms of absolute changes in mean discharge rate was minimal.

During the end of the gap ( $t 2)$ epoch, the mean discharge rate of fixation neurons for both express and regular saccades was significantly lower compared with that during visual fixation $(t 1)$ epoch (paired $t$ test, $p<0.05$ ), whereas the mean discharge rate of buildup and burst neurons during $t 2$ was significantly increased over $t 1$ (paired $t$ test, $p<0.05$ ). The mean discharge rate of 
fixation neurons (Fig. 11B) was $39 \pm 4$ (range, 15-74) spikes/sec before express saccades and $41 \pm 3$ (range, 18-80) spikes/sec before regular saccades (paired $t$ test, $d f=23 ; t=-0.61 ; p=$ $0.55)$. Among individual fixation neurons, 2 of 24 had a significant increase, and 2 of 24 had a significant decrease in discharge before express saccades $(t$ test, $p<0.05)$. In sharp contrast, all buildup neurons discharged more vigorously during the end of gap $(t 2)$ epoch before express saccades than before regular saccades (Fig. 10B), with all data points falling above the line of equality. Moreover, for 13 of 17 neurons this increase was significant ( $t$ test, $p<0.05)$. The mean discharge rate of buildup neurons (Fig. 11D) was $57 \pm 6$ (range, 20-104) spikes/sec before express saccades and $32 \pm 5$ (range, 6-73) spikes/sec before regular saccades, a significant difference (paired $t$ test, $d f=16$; $t=7.43 ; p<0.0001)$. The mean discharge rate of burst neurons during the end of gap $(t 2)$ epoch (Fig. $11 F$ ) was $14 \pm 2$ (range, $0-47$ ) spikes/sec before express saccades and $8 \pm 1$ (range, $0-33$ ) spikes/sec before regular saccades (Wilcoxon signed rank test, $p<$ $0.005)$. Thus, at the end of gap ( $t 2)$ epoch, the discharge rate of buildup neurons and, to a lesser extent, burst neurons was predictive of the mode of saccade initiation, whereas the discharge rate of fixation neurons had no predictive capability.

If the hypothesis that the level of buildup neuron activity before target appearance is predictive of the mode of saccade initiation, then the level of activity should be higher for express saccades regardless of gap duration. We therefore also used a second method to analyze the activity level of both fixation and buildup neurons for those blocks of trials in which the gap duration was randomized between 0 and $800 \mathrm{msec}$. The number of neurons yielding data sufficient for analysis (17 fixation neurons and 6 buildup neurons) was lower than for the obtained from the constant $200 \mathrm{msec}$ gap trials, because not all neurons were tested in this condition, and often the number of trials yielding express saccades was reduced. Nevertheless, the same pattern of neuronal activity with respect to mode of saccade latency was manifest; during the end of gap ( $t 2)$ epoch, buildup neuron activity best predicted the mode of saccade latency. During the visual fixation $(t 1)$ epoch there was no difference in the mean discharge rate of either class of neuron before express or regular latency saccades. The mean discharge rate \pm SEM of fixation neurons was $58 \pm 6$ (range, 22-114) spikes/sec before express saccades and $63 \pm 7$ (range, 25-134) spikes/sec before regular saccades (paired $t$ test, $d f=16 ; t=-1.56 ; p=0.14$ ). The mean discharge rate of buildup neurons was $15 \pm 5$ (range, 1-33) spikes/sec before express saccades and $12 \pm 5$ (range, 0-28) spikes/sec before regular saccades (paired $t$ test, $d f=5 ; t=0.90$; $p=0.41)$. During the end of gap ( $t 2)$ epoch, the mean discharge rate of fixation neurons was $42 \pm 5$ (range, 14-85) spikes/sec before express saccades and $44 \pm 6$ (range, 16-90) spikes/sec before regular saccades (paired $t$ test, $d f=16$; $t=-0.52$; $p=$ $0.61)$. The activity of buildup neurons during the end of gap $(t 2)$ epoch differed significantly between express and regular latency saccades. The mean discharge rate was $52 \pm 9$ (range, 27-82) spikes/sec for express saccades and $28 \pm 6$ (range, 6-46) spikes/ sec for regular saccades (paired $t$ test, $d f=5 ; t=3.49 ; p<0.05$ ). Thus, the results of the analysis of the randomized gap duration trials confirms that obtained with fixed 200 msec gap trials, namely, that the discharge rate of buildup neurons at the end of gap ( $\mathrm{t} 2$ ) epoch is the best predictor of the mode of saccade initiation.

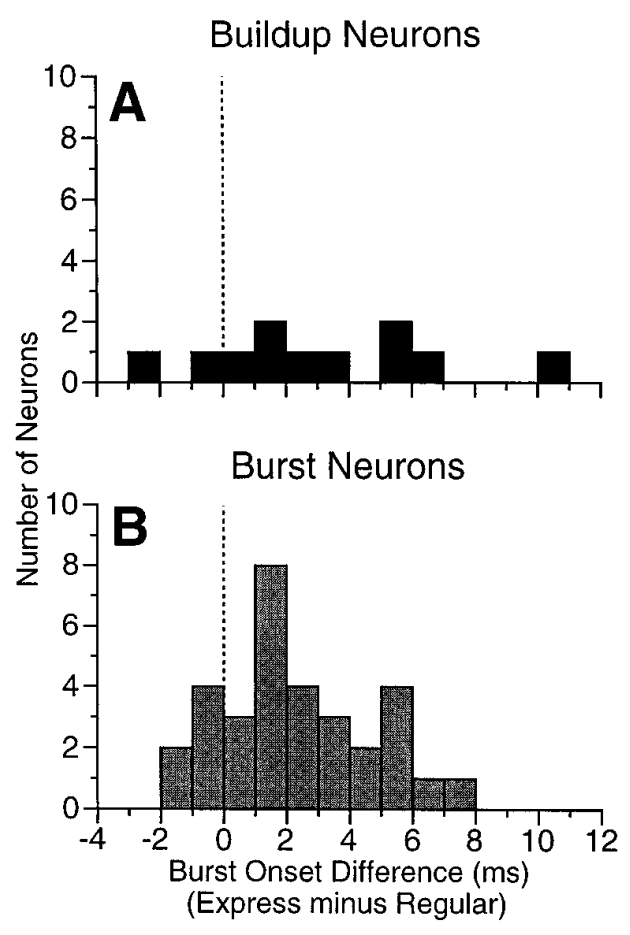

Figure 12. Difference in the target-related burst onset between express and regular saccades of buildup $(A)$ and burst $(B)$ neurons. The majority of burst and buildup neurons showed positive differences, thereby indicating that the burst occurred later after target appearance for regular than express saccades.

\section{Timing of target-related responses}

We have shown that buildup and burst neurons had a higher level of activity at the time of target presentation before the generation of express saccades. Because buildup and burst neurons with both target- and saccade-related responses for regular saccades show only one burst of action potentials for express saccades (Figs. $8 B$, $9 A$; also see Edelman and Keller, 1996), we speculated that the target-related burst of all saccade-related neurons may occur earlier for express than regular saccades. From our sample of neurons recorded for at least five trials of both express and regular saccades, 11 buildup and 32 burst neurons displayed significant target-related responses to allow for a quantitative analysis of their timing after target appearance. The majority of buildup neurons analyzed (9 of 11) were found to have an earlier time to the onset of the initial burst when the upcoming saccade was of express rather than regular latency (Fig. 12A). The mean burst onset occurred $3.1 \pm 1.1 \mathrm{msec}$ earlier for express compared with regular saccades (paired $t$ test, $d f=10 ; t=-2.79 ; p<0.02$ ). The majority of burst neurons analyzed (26 of 32) also showed earlier burst onset for express rather than regular saccades (Fig. $12 B)$. The mean burst onset occurred $2.4 \pm 0.4$ msec earlier for express compared with regular saccades (paired $t$ test, $d f=31 ; t=$ $-5.45 ; p<0.0001)$. This analysis also reveals that before target appearance there was an increase in excitability of buildup and burst neurons when an express saccade was imminent.

\section{Magnitude of target- and saccade-related responses}

It has been hypothesized that express saccades result from targetrelated responses of SC saccade-related neurons becoming the saccadic trigger signal (Sommer 1994; Edelman and Keller 1996). If this were correct, the peak of the target-related responses observed for regular saccade trials should not reach some thresh- 
Figure 13. Comparison between express (abscissa) and regular (ordinate) saccades in the magnitude of the peak of the target-aligned burst of activity $(A)$ and saccade-aligned burst of activity $(B)$ of buildup (filled squares) and burst (open triangles) neurons. $C$, Comparison between the magnitude of the peak of the target-aligned (abscissa) and saccade-aligned (ordinate) bursts for regular saccades. The dotted line represents the equality line.
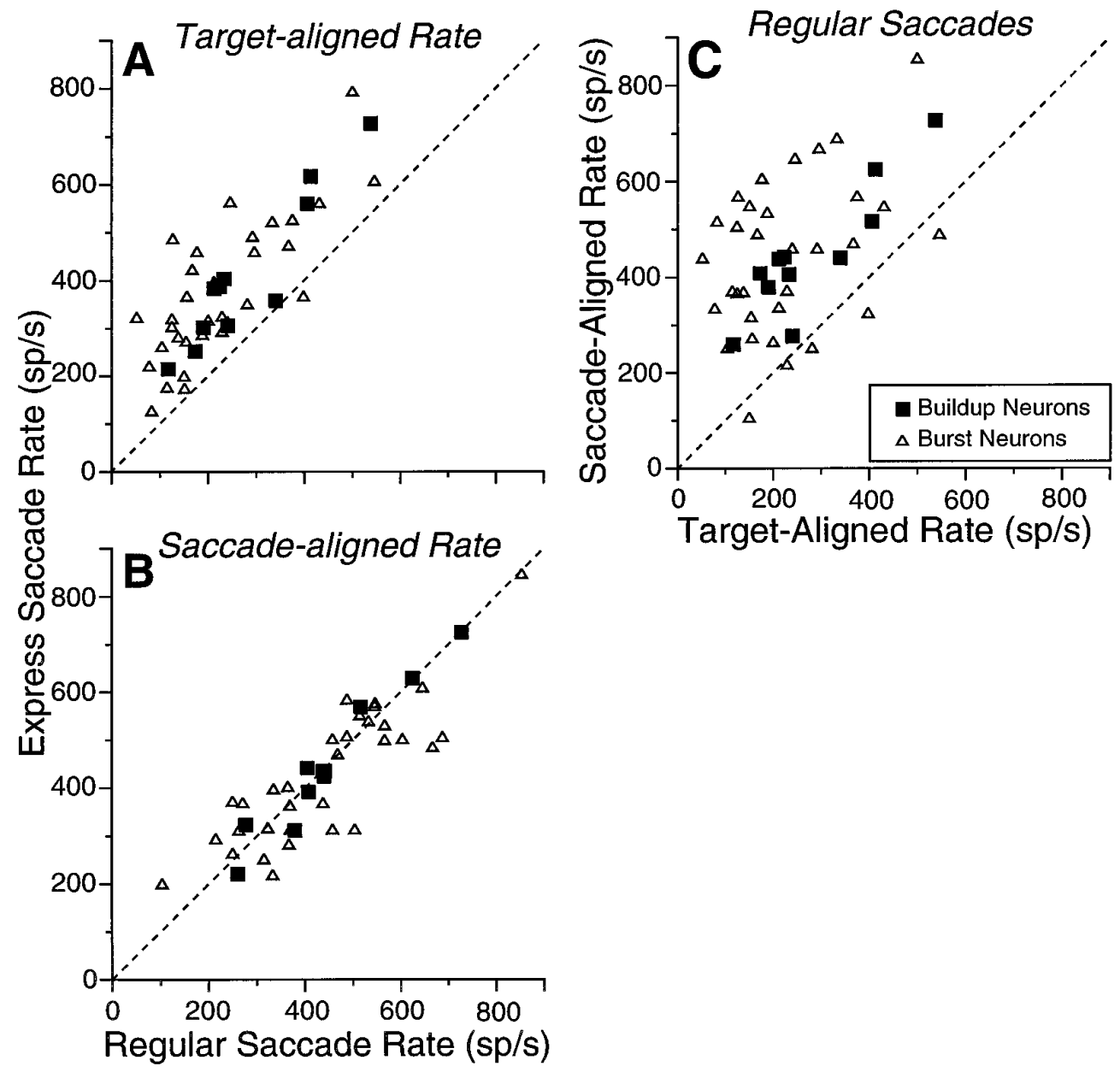

Target-Aligned Rate (sp/s) old level and therefore should always be less than the peak of the single burst of activity accompanying express saccades or the saccade-related burst of regular saccades. Figures $8 B$ and $9 A$ illustrate that for the buildup and burst neurons that displayed significant target-related responses, the target-related burst occurring in regular saccade trials was indeed consistently smaller than either the saccade-related burst or the single burst accompanying express saccades. Noteworthy, the first burst observed in regular saccade trials was clearly target-related, because it faithfully followed target appearance for all gap durations tested (not shown). In addition, the target-related burst occurred only when the target was presented in the response field of a neuron and not elsewhere in the visual field. To test the hypothesis that this target-related burst of activity represents a response that failed to achieve the saccadic trigger threshold, we measured the magnitude of these bursts of activity when the rasters and spike density functions were aligned on either target appearance or saccade onset (Fig. 13). Figure $13 A$ contrasts for express and regular saccades, the magnitude of the target-aligned burst after target appearance for the 32 burst neurons and the 11 buildup neurons that displayed significant target-related responses. For all neurons but one burst neuron, the magnitude of the target-aligned burst preceding express saccades was greater than the target-aligned burst preceding regular saccades. The mean discharge rate of the peak of the target-aligned burst for buildup neurons was $410 \pm 48$ spikes/sec before express saccades and $280 \pm 38$ spikes/sec before regular saccades. This difference was significant (paired $t$ test, $d f=10 ; t=7.3 ; p<0.0001)$. The mean peak burst rate for burst neurons was $375 \pm 26$ spikes/sec before express saccades and $227 \pm 22$ spikes/sec before regular saccades, and this difference was also highly significant (paired $t$ test, $d f=31 ; t=9.06 ; p<$ 0.0001).

Despite the significant difference in the magnitude of the target-aligned burst for express and regular saccades, there was no difference in the magnitude of the saccade-aligned bursts. Figure $13 B$ contrasts for express and regular saccades the magnitude of the saccade-aligned burst for the same burst and buildup neurons. Note that most data points scatter along the equality line, indicating that the saccade-aligned burst of express and regular saccades was of similar magnitude. For buildup neurons, the mean peak burst rate was $446 \pm 44$ spikes/sec before express saccades and $447 \pm 41$ spikes/sec before regular saccades (paired $t$ test, $d f=10 ; t=-0.09 ; p=0.93)$. For burst neurons, the mean peak burst rate was $424 \pm 25$ spikes/sec before express saccades and $443 \pm 28$ spikes/sec before regular saccades, and this difference was not significant (paired $t$ test, $d f=31 ; t=-1.21 ; p=$ 0.23 ). Therefore, the threshold required to elicit a saccade may have remained constant whether a saccade was either of express or regular latency. When compared with the saccade-aligned burst accompanying regular saccades (Fig. 13C), the magnitude of the target-aligned burst of activity was also found to be significantly smaller for the sample of burst neurons (paired $t$ test, $d f=$ $31 ; t=-7.69 ; p<0.0001$ ) and buildup neurons (paired $t$ test, $d f=$ $10 ; t=-8.86 ; p<0.0001)$. The results of this analysis reveal that, 
for both burst and buildup neurons, there was a significant difference between the magnitude of the target-aligned burst for express and regular saccades but not between the magnitude of the saccade-aligned burst.

\section{DISCUSSION}

We have shown that neuronal activity within the SC is modulated in the gap period of the gap paradigm before target presentation in a manner that could account for variation in SRT, including both the gap-related reduction in SRTs and the occurrence of express saccades. The attenuation of fixation neuron activity during the gap may represent a disengagement of ocular fixation (Reuter-Lorenz et al., 1991; Munoz and Wurtz, 1992, 1993b; Kingstone and Klein, 1993b; Sommer, 1994; Tam and Ono, 1994; Dorris and Munoz, 1995) and may lead to the global disinhibition of the saccade-generating circuitry and thus may contribute to the gap effect. However, we have shown that the activity level of fixation neurons was not correlated with SRT (see Figs. 6 $A-D, 7 A$, Tables 1, 2) or express saccade occurrence (see Figs. 8, 10,11). Rather, our data suggest that the early preparation of oculomotor programs is necessary for reduced SRTs and the occurrence of express saccades. Gap-related activity of buildup neurons was correlated to SRT most consistently when the ensuing saccade was directed to a target located in the response field of the neuron (see Figs. $6 F, 7 B$, Tables 1,2 ) and was a good predictor of whether the saccade would be of express or regular latency (see Figs. 8, 10, 11). Furthermore, the target-related responses of both burst and buildup neurons before express saccades occurred earlier (see Fig. 12) and were of a greater magnitude (see Fig. 13) than for regular saccades, indicative of a higher level of excitability. Altogether, these observations demonstrate the involvement of the SC in saccade initiation and provide strong support for the oculomotor preparation hypothesis of express saccade generation described by Paré and Munoz (1996) and derived from those of Becker (1989) and Kowler (1990).

\section{Collicular role in the gap effect and express saccade generation}

The activity of SC neurons is likely shaped by sensory, motor, and cognitive inputs (for review, see Sparks and Hartwich-Young, 1989). The important sensory signals impinging on the SC during the gap paradigm include the appearance and disappearance of visual stimuli at the fovea and eccentric locations. We argue that the gap effect and express saccades are likely the result of setting levels of excitability among populations of collicular neurons during the gap period before target presentation.

While the FP is present, both sensory (foveal visual inputs) and cognitive (the monkey's reward is contingent on foveation of the FP) signals are at their highest level, centered on fixation neurons on the SC saccadic motor map. This results in vigorous activation of fixation neurons and inhibition of saccade-related neurons in the rest of the SC through hypothesized lateral interactions (Munoz and Guitton, 1991; Munoz and Wurtz, 1993b,c, 1995b). If, at this time, a target is presented at an eccentric location, a relatively long time is required to reduce the neural activity of fixation neurons and correspondingly to increase the activity at the appropriate locus on the SC motor map.

Disappearance of the FP during the gap removes sensory inputs to fixation neurons, leading to a disinhibition of saccaderelated neurons. In this case, when the target is presented, less time is required for saccade-related neurons to generate the saccade motor command, and the SRT is reduced, the gap effect. In addition, the onset of the gap may be associated with the impending target appearance (Ross and Ross, 1981; Kingstone and Klein, 1993a) and therefore increase nonspecific preparatory inputs to buildup neurons. Such inputs may arise from frontal cortex (Dias and Bruce, 1994; Everling et al., 1996).

Although we propose that the generation of express saccades is facilitated by the reduced inhibition afforded by attenuated fixation neurons, we have shown that fixation neuron attenuation is, by itself, insufficient to account for express saccade generation. Rather, our data suggest that the increase in the activity of buildup neurons coding for the upcoming target location is necessary for express saccade generation. This localized increase in activity may be caused by inputs related to the monkey's expectation or familiarity of where the target is most likely to be presented after the gap (Paré and Munoz, 1996). This familiarity can be achieved either through exposure to a limited set of target locations or by increasing the probability of a target being presented at a certain location. When the target is ultimately presented, the active neurons at the SC locus coding for the saccade are so close to threshold that the addition of the target-related responses can trigger the saccade at an express latency.

Earlier studies by Munoz and Wurtz (1992, 1993b) suggested that the level of fixation neuron activity was crucial in the generation of express saccades. In these experiments, the injection of muscimol, an agonist of the endogenous neurotransmitter GABA, into the fixation region of the rostral SC of monkeys led to a high incidence of express saccades. We argue that the muscimol may have decreased fixation neuron activity to such a nonphysiological level that inhibition was removed from the remainder of the SC, thereby indirectly facilitating the generation of express saccades. Furthermore, the results of Munoz and Wurtz $(1992,1993 b)$ are somewhat confounded by the fact that the monkey had partial information about where the target would be presented (one of two possible locations); the monkey's familiarity with these target locations could have led to increased buildup activity at the corresponding SC loci. Interestingly, Hikosaka and Wurtz (1985) made the observation that short-latency saccades (with latency often $<100 \mathrm{msec}$ ) are initiated after injection of bicuculline (GABA antagonist) in SC sites containing saccade-related neurons. These responses occurred whenever a target was presented in the response field of the local neurons, irrespective of whether the animal had to maintain fixation. These results are consistent with our hypothesis that an increased level of preparatory activity in the SC permits reduced SRTs and ultimately express saccades.

\section{Target-related responses and SRT bimodality}

The excitability levels of fixation and buildup neurons established by sensory and cognitive inputs before target presentation fulfill predictions of mathematical models of SRT reductions during the gap paradigm (Carpenter and Williams, 1995; Kopecz, 1995; Kopecz and Schoner, 1995). However, these models predict normal distributions of SRTs and cannot account for the observed bimodality (see Fig. 2). This bimodality can be explained by incorporating the merging of the target- and saccade-related discharges of most buildup neurons (Fig. $8 B$ ) and burst neurons (Fig. 9A; Edelman and Keller, 1996) accompanying express saccades with the gap-related modulations of fixation and buildup neuron activity. Because both the target- and saccade-related discharges are simply bursts of action potentials from the same 
neuron, it has been proposed that both may act as the trigger for a saccade (Sommer, 1994; Edelman and Keller, 1996). For regular saccades, the saccade-generating circuit may be sufficiently inhibited at the time of target presentation such that the target-related burst cannot surpass the hypothetical threshold to elicit a saccade. If, however, the visual target signal impinges on a region of the SC comprising buildup neurons with sufficiently high activity, the ensuing target-related burst may surpass this threshold and trigger an express saccade. Indeed, the single burst associated with express saccades is earlier and more robust than the targetrelated response observed for regular saccade trials (see Figs. $8 B$, $9 A, 12,13 A, C)$, and its timing is equally correlated to the onset of the stimulus and the initiation of the movement (Edelman and Keller, 1996). The earlier target-aligned burst onset time we measured differs from that of Edelman and Keller (1996), who showed only a nonsignificant reduction in the timing of the burst onset for express saccades. This discrepancy may be explained by differences in the methods used to determine the burst onset or the number of neurons considered in each sample.

The necessity of the transient target-related burst of saccaderelated neurons for the generation of express saccades explains other findings particular to this class of saccades. For example, express saccades cannot be generated to targets illuminated continuously, as in the delayed saccade paradigm in which the FP disappearance is the cue to initiate the saccade (Boch and Fischer, 1986; Rohrer and Sparks, 1993; Edelman and Keller, 1996). Neither can they be directed away from targets, as in the antisaccade paradigm (Fischer and Weber, 1992), in which the transient burst is not generated at the proper location on the SC map. Only when two targets are presented in close proximity can express saccades be made to a location where no target is present, that being the average position of the two stimuli (Chou et al., 1994). In this case, the transient bursts are generated at nearby SC loci, and the respective populations of activated neurons are presumably overlapping; thus the end point of these averaging express saccades is neurally represented. Unlike the obligatory sudden appearance of a target, a temporal gap between the fixation point and the target is not required to produce express saccades (Sommer, 1994; Paré and Munoz, 1996).

\section{Early increase in activity and preparation for action}

Saccadic eye movements are not the only motor responses for which early changes in neuronal activity can be related to preparatory processes. For instance, Requin and colleagues (Lecas et al., 1986; Riehle and Requin, 1989, 1993; Requin et al., 1990) demonstrated that the level of neuronal activity in motor, premotor, parietal, and prefrontal cortex during a preparatory period is predictive of the reaction time of limb movements. In these experiments, changes in both neuronal activity and reaction time were induced by manipulating either the response probability (using a movement-priming paradigm similar to the gap paradigm) or previous information about the kinematics of the movement to be executed (using a movement-precuing paradigm). In our experiments, only spontaneous changes in SRT and neuronal activity were examined (for an analogous approach in studying reaching movements, see Kubota and Hamada, 1979), but nevertheless a significant proportion of SC buildup neurons exhibited significant correlation. It thus remains to be demonstrated whether experimental manipulations could enhance this relationship. In conclusion, the timing of initiation of motor responses appears to be intimately linked to the excitability state of neural elements distributed throughout brain structures and may reflect presetting processes commonly referred to as the preparatory set (Evarts et al., 1984).

\section{REFERENCES}

Becker W (1989) Metrics. In: The neurobiology of saccadic eye movements (Wurtz RH, Goldberg ME, eds), pp 13-67. Amsterdam: Elsevier.

Boch R, Fischer B (1986) Further observations on the occurrence of express-saccades in the monkey. Exp Brain Res 63:487-494.

Carpenter RHS (1981) Oculomotor procrastination. In: Eye movements: cognition and visual perception (Fischer DF, Monty RA, eds), pp 237-246. Hillsdale, NJ: Erlbaum.

Carpenter RHS, Williams MLL (1995) Neural computation of log likelihood in control of saccadic eye movements. Nature 377:59-62.

Chou I, Sommer MA, Schiller PH (1994) Bimodal latency distribution of averaging saccades in monkey. Soc Neurosci Abstr 20:1401.

Crist CF, Yamasaki DSG, Komatsu H, Wurtz RH (1988) A grid system and a microsyringe for single cell recording. J Neurosci Methods 26:117-122.

Dias EC, Bruce CJ (1994) Physiological correlate of fixation disengagement in the primate's frontal eye field. J Neurophysiol 72:2532-2537.

Dorris MC, Munoz DP (1995) A neural correlate for the gap effect on saccadic reaction times in monkey. J Neurophysiol 73:2558-2562.

Dorris MC, Paré M, Istvan PJ, Lablans A, Munoz DP (1995) Saccadic reaction times in the monkey. II. Neural correlates in the superior colliculus. Soc Neurosci Abstr 21:1193.

Edelman JA, Keller EL (1996) Activity of visuomotor burst neurons in the superior colliculus accompanying express saccades. J Neurophysiol 76:908-926.

Evarts EV, Shinoda Y, Wise SP (1984) Neurophysiological approaches to higher brain functions. New York: Wiley.

Everling S, Krappmann P, Spantekow A, Flohr H (1996) Cortical potentials during the gap before express saccades and fast regular saccades. Exp Brain Res 111:139-143.

Fischer B, Boch R (1983) Saccadic eye movements after extremely short reaction times in the monkey. Brain Res 260:21-26.

Fischer B, Ramsperger E (1984) Human express saccades: extremely short reaction times of goal directed eye movements. Exp Brain Res 57:191-195.

Fischer B, Weber H (1992) Characteristics of "anti" saccades in man. Exp Brain Res 89:415-424.

Fischer B, Weber H (1993) Express saccades and visual attention. Behav Brain Sci 16:553-610.

Fischer B, Boch R, Ramsperger E (1984) Express-saccades of the monkey: effect of daily training on probability of occurrence and reaction time. Exp Brain Res 55:232-242.

Fuchs AF, Robinson DA (1966) A method for measuring horizontal and vertical eye movement chronically in the monkey. J Appl Physiol 21:1068-1070.

Hanes DP, Thompson KG, Schall JD (1995) Relationship of presaccadic activity in frontal eye field and supplementary eye field to saccade initiation in macaque: Poisson spike train analysis. Exp Brain Res 103:85-86.

Hays AV, Richmond BJ, Optician LM (1982) A UNIX-based multiple process system for real-time data acquisition and control. W ESCON Conf Proc 2:1-10.

Hikosaka O, Wurtz RH (1985) Modification of saccadic eye movements by GABA-related substances. I. Effect of muscimol and bicuculline in monkey superior colliculus. J Neurophysiol 53:266-291.

Hikosaka O, Wurtz RH (1986) Saccadic eye movements following injection of lidocaine into the superior colliculus. Exp Brain Res 61:531-539.

Judge SJ, Richmond BJ, Chu FC (1980) Implantation of magnetic search coils for measurement of eye position: an improved method. Vision Res 20:535-538.

Kingstone A, Klein RM (1993a) What are human express saccades? Percept Psychophys 54:260-273.

Kingstone A, Klein RM (1993b) Visual offsets facilitate saccadic latency: does predisengagement of visuospatial attention mediate this gap effect? J Exp Psychol 19:1251-1265.

Kopecz K (1995) Saccadic reaction times in gap/overlap paradigms: a model based on integration of intentional and visual information on neural, dynamic fields. Vision Res 35:2911-2925.

Kopecz K, Schoner G (1995) Saccadic motor planning by integrating visual information and pre-information on neural dynamic fields. Biol Cybern 73:49-60. 
Kowler E (1990) The role of visual and cognitive processes in the control of eye movements. In: Eye movements and their role in visual and cognitive processes, reviews of oculomotor research (Kowler E, ed), pp 353-393. Amsterdam: Elsevier.

Kubota K, Hamada I (1979) Preparatory activity of monkey pyramidal tract neurons related to quick movement onset during visual tracking performance. Brain Res 168:435-439.

Lecas J-C, Requin J, Anger C, Vitton N (1986) Changes in neuronal activity of the monkey precentral cortex during preparation for movement. J Neurophysiol 56:1680-1702.

MacPherson JM, Aldridge JW (1979) A quantitative method of computer analysis of spike train data collected from behaving animals. Brain Res 175:183-187.

Munoz DP, Guitton D (1991) Control of orienting gaze shifts by the tectoreticulospinal system in the head-free cat. II. Sustained discharges during motor preparation and fixation. J Neurophysiol 66:1624-1641.

Munoz DP, Wurtz RH (1992) Role of the rostral superior colliculus in active visual fixation and execution of express saccades. J Neurophysiol 67:1000-1002.

Munoz DP, Wurtz RH (1993a) Fixation cells in monkey superior colliculus. I. Characteristics of cell discharge. J Neurophysiol 70:559-575.

Munoz DP, Wurtz RH (1993b) Fixation cells in monkey superior colliculus. II. Reversible activation and deactivation. J Neurophysiol 70:576-589.

Munoz DP, Wurtz RH (1993c) Interactions between fixation and saccade neurons in primate superior colliculus. Soc Neurosci Abstr 19:787.

Munoz DP, Wurtz RH (1995a) Saccade-related activity in monkey superior colliculus. I. Characteristics of burst and buildup cells. J Neurophysiol 73:2313-2333.

Munoz DP, Wurtz RH (1995b) Saccade-related activity in monkey superior colliculus. II. Spread of activity during saccades. J Neurophysiol 73:2334-2348.

Paré M, Munoz DP (1996) Saccadic reaction time in the monkey: advanced preparation of oculomotor programs is primarily responsible for express saccades occurrence. J Neurophysiol 76:3666-3681.

Paré M, Crommelinck M, Guitton D (1994) Gaze shifts evoked by stimulation of the superior colliculus in the head-free cat conform to the motor map but also depend on stimulus strength and fixation activity. Exp Brain Res 101:123-139.

Requin J, Lecas J-C, Vitton N (1990) A comparison of preparationrelated neuronal activity changes in the prefrontal, premotor, primary motor and posterior parietal areas of the monkey cortex: preliminary results. Neurosci Lett 111:151-156.

Reuter-Lorenz PA, Hughes HC, Fendrich R (1991) The reduction of saccadic latency by prior offset of the fixation point: an analysis of the gap effect. Percept Psychophys 49:167-175.
Richmond BJ, Optician LM, Podell M, Spitzer H (1987) Temporal encoding of two-dimensional patterns by single units in primate inferior temporal cortex. I. Response characteristics. J Neurophysiol 57:132-146.

Riehle A, Requin J (1989) Monkey primary motor and premotor cortex: single-cell activity related to prior information about direction and extent of an intended movement. J Neurophysiol 61:534-549.

Riehle A, Requin J (1993) The predictive value for performance speed of preparatory changes in neuronal activity of the monkey motor and premotor cortex. Behav Brain Sci 53:35-49.

Rohrer WH, Sparks DL (1993) Express saccades: the effects of spatial and temporal uncertainty. Vision Res 33:2447-2460.

Ross SM, Ross LE (1981) Saccadic latency and warning signals: effects of auditory and visual stimulus onset and offset. Percept Psychophys 29:429-437.

Schiller PH, Sandel JH, Maunsell JHR (1987) The effect of frontal eye field and superior colliculus lesions on saccadic latencies in the rhesus monkey. J Neurophysiol 57:1033-1049.

Sommer MA (1994) Express saccades elicited during visual scan in the monkey. Vision Res 34:2023-2038.

Sparks DL (1978) Functional properties of neurons in the monkey superior colliculus: coupling of neuronal activity and saccade onset. Brain Res 156:1-16.

Sparks DL, Hartwich-Young R (1989) The deep layers of the superior colliculus. In: The neurobiology of saccadic eye movements (Wurtz RH, Goldberg ME, eds), pp 213-255. Amsterdam: Elsevier.

Sparks DL, Holland R, Guthrie BL (1976) Size and distribution of movement fields in the monkey superior colliculus. Brain Res 113:21-34.

Stanford TR, Freedman EG, Sparks DL (1996) Site and parameters of microstimulation: evidence for independent effects on the properties of saccades evoked from the primate superior colliculus. J Neurophysiol 76:3360-3381.

Tam WJ, Ono H (1994) Fixation disengagement and eye-movement latency. Percept Psychophys 56:251-260.

Thompson KG, Hanes DP, Bichot NP, Schall JD (1996) Perceptual and motor processing stages identified in the activity of macaque frontal eye field neurons during visual search. J Neurophysiol 76:4040-4055.

Waitzman DM, Ma TP, Optician LM, Wurtz RH (1991) Superior colliculus neurons mediate the dynamic characteristics of saccades. J Neurophysiol 66:1716-1737.

Wurtz RH, Goldberg ME (1972) Activity of superior colliculus in behaving monkey. III. Cells discharging before eye movements. J Neurophysiol 35:575-586. 